\title{
Tracking modal interactions in nonlinear energy sink dynamics via high-dimensional invariant manifold
}

\author{
Giuseppe Habib • Francesco Romeo
}

Received: 1 March 2020 / Accepted: 1 September 2020 / Published online: 15 September 2020

(C) The Author(s) 2020

\begin{abstract}
A nonlinear energy sink (NES), conceived to mitigate the vibrations of a multi-degree-of-freedom host mechanical system, is considered. The highdimensional slow invariant manifold (SIM) describing the high-amplitude slow dynamics of the system is derived and exploited to interpret its transient regimes caused by impulsive excitation. It is shown that algebraic expressions derived from the SIM formulation enable to identify the so-called interaction points, providing the conditions in which two modes of the primary system interact and share energy through the nonlinear absorber. Moreover, the mutual effect of differently activated host system modes on the NES energy dissipation mechanism is discussed. Through sections of the multidimensional SIM, modal interaction triggering resonance capture cascades (RCC) can be effectively explained. The dissipation capabilities are eventually assessed in order to evaluate the efficiency of the RCC regime.
\end{abstract}

Keywords Nonlinear energy sink · Nonlinear vibration absorber . Slow invariant manifold . Impulsive dynamics · Vibration mitigation

\section{G. Habib ( $₫)$}

Department of Applied Mechanics, MTA-BME Lendület Human Balancing Research Group, Budapest University of Technology and Economics, Budapest, Hungary

e-mail: habib@mm.bme.hu

F. Romeo

Department of Structural and Geotechnics Engineering,

Sapienza University of Rome, Rome, Italy

\section{Introduction}

The mitigation of impact-induced vibrations in structural systems represents a challenging task in a variety of engineering contexts. Irrespective of the specific application, a realistic representation of such systems implies to consider either continuous or multi-degreeof-freedom mechanical (MDOF) models. So, the mitigation task is accomplished by absorbing efficiently energy from main excited systems, the dynamics of which typically involve either infinite or large number of frequencies. Thus, from an engineering standpoint, the goal is to devise the minimum number of possibly passive vibration absorbers designed to resonate for broad frequency band. Toward this goal, nonlinear vibration absorbers have received growing attention in the past two decades for their potentialities in overcoming the narrow frequency-band capabilities of their linear counterparts, i.e., the tuned mass damper (i.e., TMD). Within this context, the nonlinear energy sink (NES), consisting of a small mass connected to the primary system by an essential nonlinear spring, has been extensively studied. As shown in a series of works [1,2], the lack of any preferential resonance frequency of the NES makes it capable of resonating with any mode of the primary structure. In the case of multimodal response, resonance capture cascades (RCC) during which the nonlinear attachment resonates with different modes sequentially, from higher to lower ones, were also observed [3-7]. In these studies, the transient dynamics following the impulsive excitation applied 
to the primary system are mostly investigated by considering specific manifolds, namely the nonlinear normal modes (NNM) and the impulsive orbits (IO), in the background of the underlying Hamiltonian system. Accordingly, the energy redistribution modalities are discussed by varying the initial excitation in terms of energy level and position in the primary system. The ensuing temporal evolution of the energy redistribution within the system, including RCC, is interpreted by means of frequency energy plots. Suitable measures of absorption efficiency are introduced in order to assess and possibly exploit the most favorable dynamic regimes [8].

From the analytical standpoint, different asymptotic approaches enabling to reduce the dimension of nonlinear systems comprising NESs have been so far proposed; given the usual assumption on the NES, i.e., small mass and nonlinearizable stiffness, they were mostly based on singular perturbations and invariant manifold considerations. Irrespective of the chosen asymptotic approach, the slow invariant manifold (SIM), i.e., the set of fixed points for amplitude of oscillations for the fast timescale, has been abundantly resorted to. It enabled to interpret the main dynamic regimes characterizing the nonlinear response of different types of NESs under either impulsive or harmonic excitation [8]; for different physical systems, interesting dynamic regimes and their connection to the SIM topological features have been highlighted [9].

Set of NESs were recently proposed for passive mitigation of dynamic instability in discrete systems [10,11]. Nonlinear SDOF and MDOF primary systems were considered, respectively, providing the socalled mitigation limit of the NESs, i.e., the value of a chosen bifurcation parameter which separates harmful situations from harmless situations. To this end, an asymptotic analysis of the slow flow based on geometric singular perturbation theory was adopted showing that the critical manifold of the system can be reduced to a one-dimensional parametric curve evolving in a multidimensional space. In [12], the passive control of a two-degree-of-freedom pendulum by a NES with cubic restoring force was investigated resorting to the phase-dependent SIM derived from complexification and multiple-scale methods.

In further recent studies [13,14], the RCC phenomenon was again addressed in order to guide NES design by identifying a tuning procedure and predicting the so-called cascading time. The latter studies hinge on the single-mode SIM which is derived for the general case of NES stiffness (odd) polynomial nonlinearity and small positive or negative linear part. For multimodal vibrations and RCC, the proposed algebraic performance measures are extended, albeit based on the single-mode assumption, by introducing a modal decomposition of the initial conditions.

Recently, the use of the SIM was proposed by the authors to design a tuned bistable NES, a nonlinear attachment capable of mitigating efficiently oscillations of the primary system at different energy levels and for more than one mode of vibration [15]. Further studies addressed qualitative and quantitative comparisons between TMD and NES capabilities for mitigation of broadband impulsive energy; by exploiting the four-dimensional invariant manifold of a two-DoF host system, an effective performance measure was introduced, the so-called relative dissipation power [16]. Stemming from the latter study, NES connected to a multi-degree-of-freedom (MDOF) system is here considered. Invariant manifolds describing the high-amplitude slow dynamics are analytically identified providing useful algebraic equations. These highdimensional surfaces, which relate the absorber vibration amplitude to the primary system ones, allow to explain the involved modal interaction governing the temporal evolution of the energy redistribution associated with the presence of the nonlinear absorber. It is worth emphasizing that the present study tackles the dissipation mechanism analysis with reference to the linear modes of the primary system. Differently from what is mostly reported in the literature, it is shown that such modes enable to explain the main vibration mitigation phenomena triggered by the NES.

The paper is organized as follows. At first, in Sect. 2, the mechanical model and the dimensionless equations of motion are presented to pave the way to the slow invariant manifold derivation described in Sect. 3. In Sect. 4, the SIM analysis, including its stability, is carried out aiming at identifying the conditions in which vibration energy is shared by more than one mode of the primary system. Moreover, the mutual effect of differently activated host system modes on the NES energy dissipation mechanism is discussed. In Sect. 5, the modal interaction triggering resonance capture cascades is explained by means of suitable sections of the multidimensional SIM. Section 6 is devoted to assess the dissipation performance corresponding to $\mathrm{RCC}$ by comparing the primary system energy decre- 
ment for different dynamic regimes. The conclusive section summarizes the main findings and points out open questions worth to be further investigated.

\section{Mechanical model and non-dimensionalization}

The dynamics of an NES attached to an undamped linear $n$-DOF primary system are modeled by the following system of differential equations

$$
\begin{aligned}
& \sum_{j=1}^{n} m_{h j} \ddot{x}_{j}+\sum_{j=1}^{n} k_{h j} x_{j}=0 \text { for } h=1, \ldots, n, h \neq l \\
& \sum_{j=1}^{n} m_{l j} \ddot{x}_{j}+\sum_{j=1}^{n} k_{l j} x_{j}+c_{\mathrm{a}}\left(\dot{x}_{l}-\dot{x}_{n+1}\right) \\
& \quad+k_{\mathrm{nl}}\left(x_{l}-x_{n+1}\right)^{3}=0 \\
& \varepsilon \ddot{x}_{n+1}+c_{\mathrm{a}}\left(\dot{x}_{n+1}-\dot{x}_{l}\right)+k_{\mathrm{nl}}\left(x_{n+1}-x_{l}\right)^{3}=0, \quad(1)
\end{aligned}
$$

where $m_{h j}=m_{j h}$ and $k_{h j}=k_{j h}$ are the terms of the primary system mass and stiffness matrices, $\varepsilon$ is the absorber mass, $c_{\mathrm{a}}$ and $k_{\mathrm{nl}}$ are the absorber linear damping and cubic stiffness coefficients, respectively. $\varepsilon$ is assumed small with respect to the primary system masses. We notice that the absorber is assumed to directly interact only with the $l^{\text {th }}$ DoF of the primary system. It is also assumed that the natural frequencies of the primary system are incommensurate and remote. Thus, the special case of internal resonances is not considered in this study.

In order to perform a modal analysis of the primary system, we temporarily neglect the contribution of the absorber, reducing the system to

$$
\mathbf{M} \ddot{\mathbf{x}}+\mathbf{K x}=\mathbf{0},
$$

where $\mathbf{M}$ and $\mathbf{K}$ are $n \times n$ matrices (the full system has dimension $n+1)$. Performing a classical modal analysis, we decouple the primary system, adopting the transformation $\mathbf{x}=\mathbf{U q}$, where $\mathbf{U}$ contains the eigenvalues of $\mathbf{M}^{-1} \mathbf{K}$, normalized such that $\mathbf{U}^{\mathrm{T}} \mathbf{M U}=\mathbf{I}$, where $\mathbf{I}$ is the identity matrix. $\mathbf{U}$ is defined as

$\mathbf{U}=\left[\begin{array}{ccc}u_{11} & \cdots & u_{1 n} \\ \vdots & \ddots & \vdots \\ u_{n 1} & \cdots & u_{n n}\end{array}\right]$

After modal analysis, the primary system can be rewritten as
$\ddot{\mathbf{q}}+\boldsymbol{\Omega} \mathbf{q}=\mathbf{0}$,

where $\boldsymbol{\Omega}=\left[\omega_{h}^{2}\right]$ is a diagonal matrix formed by the squares of the natural frequencies of the primary system.

By reintroducing the absorber into the system, by considering the coordinate transformation and that the NES is attached to the $l^{\text {th }}$ DoF of the primary system, we obtain the system of differential equations

$\ddot{q}_{h}+\omega_{h}^{2} q_{i}=-u_{l h}\left(c_{\mathrm{a}} \dot{z}+k_{\mathrm{n} 1} z^{3}\right)$ for $h=1, \ldots, n$

$\varepsilon \ddot{z}+c_{\mathrm{a}} \dot{z}+k_{\mathrm{nl}} z^{3}=\varepsilon \sum_{j=1}^{n} u_{l j} \ddot{q}_{j}$,

where $z=x_{l}-x_{n+1}$. Next, we introduce dimensionless absorber parameters $\zeta_{\mathrm{a}}=c_{\mathrm{a}} /\left(2 \varepsilon \omega_{1}\right)$ and $\lambda_{3}=k_{\mathrm{nl}} / \varepsilon$, attaining

$$
\begin{aligned}
& \ddot{q}_{h}+\omega_{h}^{2} q_{h}=-u_{l h} \varepsilon\left(2 \zeta_{\mathrm{a}} \omega_{1} \dot{z}+\lambda_{3} z^{3}\right) \\
& \quad \text { for } h=1, \ldots, n \\
& \ddot{z}+2 \zeta_{\mathrm{a}} \omega_{1} \dot{z}+\lambda_{3} z^{3}=\sum_{j=1}^{n} u_{l j} \ddot{q}_{j},
\end{aligned}
$$

Finally, we scale displacements with respect to the absorber's nonlinear stiffness coefficient, by introducing the coordinates $y_{h}=\sqrt{\lambda_{3}} q_{h}$ and $y_{n+1}=\sqrt{\lambda_{3}} z$. This leads to the dimensionless (in amplitude, not in time) equations of motion

$$
\begin{aligned}
& \ddot{y}_{h}+\omega_{h}^{2} y_{h}=-u_{l h} \varepsilon\left(2 \zeta_{\mathrm{a}} \omega_{1} \dot{y}_{n+1}+y_{n+1}^{3}\right) \\
& \quad \text { for } h=1, \ldots, n \\
& \ddot{y}_{n+1}+2 \zeta_{\mathrm{a}} \omega_{1} \dot{y}_{n+1}+y_{n+1}^{3}=\sum_{j=1}^{n} u_{l j} \ddot{y}_{j} .
\end{aligned}
$$

\section{Slow invariant manifold}

Aiming at characterizing the behavior of the NES against impulsive excitation, we seek for the SIM describing the slow dynamics of the system, following the procedure adopted in [15]. According to engineering practice, we assume that $\varepsilon$ is a small parameter $(\varepsilon \ll 1)$. We also assume that $q_{j}$ (for $\left.j=1, \ldots, n+1\right)$ 
are of order $\varepsilon^{1}$, while $k_{\mathrm{nl}}$ is of order $\varepsilon^{-1}$, which makes $y_{j}$ (for $j=1, \ldots, n+1$ ) having order $\varepsilon^{0}$. Additionally, according to the parameter values used in the following, we assume that $\zeta_{a}$ has order of magnitude $\varepsilon^{1 / 2}$. Neglecting terms having order of $\varepsilon$ equal to 1 or larger, we reduce the system of equations to

$\ddot{y}_{h}+\omega_{h}^{2} y_{h}=0$ for $h=1, \ldots, n$

$\ddot{y}_{n+1}+2 \zeta_{\mathrm{a}} \omega_{1} \dot{y}_{n+1}+y_{n+1}^{3}=\sum_{j=1}^{n} u_{l j} \ddot{y}_{j}$.

The system in Eq. (8) corresponds to $n$ decoupled undamped linear oscillators and one damped essentially nonlinear oscillator, excited by the other linear oscillators. The contribution of each oscillator to forcing the nonlinear one depends on its acceleration, on the modal shapes of the primary system and on its position, characterized by $u_{l j}$. With respect to energy absorption, the larger the vibration amplitude of $y_{n+1}$, the higher the dissipation power of the absorber, which is given by

$P=2 \zeta_{\mathrm{a}} \varepsilon \omega_{1} \dot{y}_{n+1}^{2}$.

We define an approximate solution by adopting the harmonic balance method, assuming 1:1 resonance between the primary system and the absorber. The solution of the first $n$ equations of (8) is given by

$y_{h}=A_{h} e^{i \omega_{h} T}+$ c.c. for $h=1, \ldots, n$,

where $A_{h}$ is complex and c.c. stands for complex conjugate. The approximate solution for $y_{n+1}$ is expressed by

$y_{n+1} \approx \sum_{j=1}^{n} B_{j} e^{i \omega_{j} T}+$ c.c.

We notice that for the case in which two or more modes are closely spaced, Eq. (11) is no longer applicable and intermodal energy exchange mediated by the NES occurs [17].

Substituting $y_{h}, h=1, \ldots, n$ into the last of Eq. (8) and collecting the different harmonics (assuming that there are no internal resonances), we have

$$
\begin{aligned}
& \left(e^{i \omega_{h} T}\right): \quad-\omega_{h}^{2} B_{h}+u_{l h} \omega_{h}^{2} A_{h} \\
& -3 B_{h}^{2} \bar{B}_{h}+6 B_{h} \sum_{j=1}^{n} B_{j} \bar{B}_{j} \\
& \quad+2 \zeta_{\mathrm{a}} \omega_{1} \omega_{h} i B_{h} \text { for } h=1, \ldots, n .
\end{aligned}
$$

By defining $A_{h}=a_{h} e^{i \alpha_{h}} / 2$ and $B_{h}=b_{h} e^{i \beta_{h}} / 2$, separating real and imaginary parts of a generic equation of (12), we have

$$
\begin{aligned}
\frac{1}{2} u_{l h} \omega_{h}^{2} a_{h} \cos \alpha_{h}= & \frac{1}{2} b_{h}\left(\omega_{h}^{2}+\frac{3}{4} b_{h}^{2}-\frac{3}{2} \sum_{j=1}^{n} b_{j}^{2}\right) \\
& \cos \beta_{h}+\zeta_{\mathrm{a}} \omega_{1} \omega_{h} b_{h} \sin \beta_{h} \\
\frac{1}{2} u_{l h} \omega_{h}^{2} a_{h} \sin \alpha_{h}= & \frac{1}{2} b_{h}\left(\omega_{h}^{2}+\frac{3}{4} b_{h}^{2}-\frac{3}{2} \sum_{j=1}^{n} b_{j}^{2}\right) \\
& \sin \beta_{h}-\zeta_{\mathrm{a}} \omega_{1} \omega_{h} b_{h} \cos \beta_{h} \\
& \text { for } h=1, \ldots, n .
\end{aligned}
$$

We calculate the squares of the two equations of (13) and sum them up attaining

$$
\begin{aligned}
u_{l h}^{2} \omega_{h}^{4} a_{h}^{2}=b_{h}^{2} & \left(\left(\omega_{h}^{2}+\frac{3}{4} b_{h}^{2}-\frac{3}{2} \sum_{j=1}^{n} b_{j}^{2}\right)^{2}\right. \\
& \left.+4 \zeta_{\mathrm{a}}^{2} \omega_{1}^{2} \omega_{h}^{2}\right) \text { for } h=1, \ldots, n,
\end{aligned}
$$

which form a system of algebraic equations which defines the SIM of the system. $a_{h}$ indicates the amplitude of oscillation of the $h^{\text {th }}$ mode of the primary system, while $b_{h}$ indicates the component of the relative amplitude of oscillation of the absorber at $\omega_{h}$ angular frequency. Equation (14) explicitly provides $a_{h}$ as a function of $b_{h}$; however, in general, it is more interesting to know the oscillation amplitude of the absorber with respect to the primary system and not the opposite. Since Eq. (14) cannot be made explicit with respect to $b_{h}$, the algebraic system of equations is numerically solved.

Without loss of generality, in the remainder of the paper a four-DoF primary system will be considered. It will allow to interpret the numerical evidence of dissipative mechanisms in the background of suitable sections of the SIM and manageable algebraic expressions.

\section{Study of the manifold}

A system consisting of four identical masses attached in a chain through five identical springs is considered. The masses can move only horizontally, and the absorber is attached to the second mass. The equations of motion of the system read 


$$
\begin{aligned}
& m \ddot{x}_{1}+2 k x_{1}-k x_{2}=0 \\
& m \ddot{x}_{2}-k x_{1}+2 k x_{2}-k x_{3}+c_{\mathrm{a}}\left(\dot{x}_{2}-\dot{x}_{5}\right) \\
& \quad+k_{\mathrm{nl}}\left(x_{2}-x_{5}\right)^{3}=0 \\
& m \ddot{x}_{3}-k x_{2}+2 k x_{3}-k x_{4}=0 \\
& m \ddot{x}_{4}-k x_{3}+2 k x_{4}=0 \\
& m_{\mathrm{a}} \ddot{x}_{5}+c_{\mathrm{a}}\left(\dot{x}_{5}-\dot{x}_{2}\right)+k_{\mathrm{nl}}\left(x_{5}-x_{2}\right)^{3}=0 .
\end{aligned}
$$

Non-dimensionalizing the system and introducing modal coordinates Eqs. (15) take the form

$$
\begin{aligned}
& \ddot{y}_{1}+\omega_{1}^{2} y_{1}=-u_{21} \varepsilon\left(2 \zeta_{\mathrm{a}} \omega_{1} \dot{y}_{5}+y_{5}^{3}\right) \\
& \ddot{y}_{2}+\omega_{2}^{2} y_{2}=-u_{22} \varepsilon\left(2 \zeta_{\mathrm{a}} \omega_{1} \dot{y}_{5}+y_{5}^{3}\right) \\
& \ddot{y}_{3}+\omega_{3}^{2} y_{3}=-u_{23} \varepsilon\left(2 \zeta_{\mathrm{a}} \omega_{1} \dot{y}_{5}+y_{5}^{3}\right) \\
& \ddot{y}_{4}+\omega_{4}^{2} y_{4}=-u_{24} \varepsilon\left(2 \zeta_{\mathrm{a}} \omega_{1} \dot{y}_{5}+y_{5}^{3}\right) \\
& \ddot{y}_{5}+2 \zeta_{\mathrm{a}} \omega_{1} \dot{y}_{5}+y_{5}^{3}=u_{21} \ddot{y}_{1}+u_{22} \ddot{y}_{2}+u_{23} \ddot{y}_{3}+u_{24} \ddot{y}_{4},
\end{aligned}
$$

where dimensionless time is $T=t \sqrt{m / k}$, amplitude is non-dimensionalized according to $\tilde{x}_{i}=x_{i} \sqrt{k_{\mathrm{nl}} /(k \varepsilon)}$ for $i=1, \ldots, 5$, with $\tilde{x}_{i}$ general dimensionless amplitude, $\varepsilon=m_{\mathrm{a}} / m$ is the mass ratio, $\zeta_{\mathrm{a}}=$ $c_{\mathrm{a}} /\left(2 \omega_{1} \varepsilon \sqrt{\mathrm{km}}\right), y_{1}, y_{2}, y_{3}$ and $y_{4}$ are the primary system dimensionless modal coordinates, $y_{5}=$ $\left(x_{2}-x_{5}\right) \sqrt{k_{\mathrm{nl}} /(k \varepsilon)}$ is the dimensionless relative displacement of the absorber with respect to the second mass, $\omega_{1}=0.618, \omega_{2}=1.1756, \omega_{3}=1.618$ and $\omega_{4}=1.9021$ are the dimensionless natural frequencies, while $u_{21}=0.6015, u_{22}=-0.3717, u_{23}=$ 0.3717 and $u_{24}=0.6015$ are the modal displacement components of the second mass, which is connected to the NES. The values of parameters $\varepsilon$ and $\zeta_{\mathrm{a}}$ are 0.02 and 0.1, respectively. Considering Eq. (14), the SIM now is given by

$$
\begin{aligned}
& u_{21}^{2} \omega_{1}^{4} a_{1}^{2}=b_{1}^{2}\left(\left(\omega_{1}^{2}-\frac{3}{4} b_{1}^{2}-\frac{3}{2} b_{2}^{2}-\frac{3}{2} b_{3}^{2}-\frac{3}{2} b_{4}^{2}\right)^{2}\right. \\
& \left.+4 \zeta_{\mathrm{a}}^{2} \omega_{1}^{2} \omega_{1}^{2}\right) \\
& u_{22}^{2} \omega_{2}^{4} a_{2}^{2}=b_{2}^{2}\left(\left(\omega_{2}^{2}-\frac{3}{4} b_{2}^{2}-\frac{3}{2} b_{1}^{2}-\frac{3}{2} b_{3}^{2}-\frac{3}{2} b_{4}^{2}\right)^{2}\right. \\
& \left.+4 \zeta_{\mathrm{a}}^{2} \omega_{1}^{2} \omega_{2}^{2}\right)
\end{aligned}
$$

$$
\begin{aligned}
& u_{23}^{2} \omega_{3}^{4} a_{3}^{2}=b_{3}^{2}\left(\left(\omega_{3}^{2}-\frac{3}{4} b_{3}^{2}-\frac{3}{2} b_{1}^{2}-\frac{3}{2} b_{2}^{2}-\frac{3}{2} b_{4}^{2}\right)^{2}\right. \\
& \left.+4 \zeta_{\mathrm{a}}^{2} \omega_{1}^{2} \omega_{3}^{2}\right) \\
& u_{24}^{2} \omega_{4}^{4} a_{4}^{2}=b_{4}^{2}\left(\left(\omega_{4}^{2}-\frac{3}{4} b_{4}^{2}-\frac{3}{2} b_{1}^{2}-\frac{3}{2} b_{2}^{2}-\frac{3}{2} b_{3}^{2}\right)^{2}\right. \\
& \left.+4 \zeta_{\mathrm{a}}^{2} \omega_{1}^{2} \omega_{4}^{2}\right) .
\end{aligned}
$$

If only one mode of the primary system is activated, the dynamics of the system can be approximately reduced to that of a single DoF with an attached NES. This case was extensively studied in the literature with similar techniques $[13-16,18]$; therefore, here we only recall its main features. Figure 1 illustrates the four different manifolds (which are actually sections of the SIM), each one represented in the $a_{h}, b_{h}$ space, for $a_{j}=0$ if $j \neq h$ (i.e., the other modes are assumed null). The curves in the figure relate the amplitude of oscillation of the absorber to that of the primary system for a specific vibration mode. Solid and dashed lines indicate stable and unstable branches, respectively; stability was studied adopting the Hill's method [19], whose direct implementation is clearly explained in [20,21]. Despite the simplicity of the procedure for obtaining these curves, they are very informative regarding the slow dynamics of the system. They can be easily related to the energy dissipated by the NES [16], and they allow to predict the time required to dissipate a certain amount of energy [13], enabling a relatively precise design of the NES, without requiring extensive numerical simulations [18].

From the SIM, it is possible to estimate the dissipation power of the absorber, which is given by Eq. (9). Taking into account only the principal harmonics of the system and integrating over one period, the average dissipation power is approximated by $P_{\mathrm{av}}=$ $\omega_{1} \varepsilon \zeta_{\mathrm{a}}\left(\omega_{1}^{2} b_{1}^{2}+\omega_{2}^{2} b_{2}^{2}+\omega_{3}^{2} b_{3}^{2}+\omega_{4}^{2} b_{4}^{2}\right)$. Then, in order to have an engineering-relevant measure of the NES performance, the average dissipation power can be divided by the energy in the primary system, obtaining what we define as relative dissipation power [16]

$$
P_{\mathrm{r}}=2 \omega_{1} \varepsilon \zeta_{\mathrm{a}} \frac{\omega_{1}^{2} b_{1}^{2}+\omega_{2}^{2} b_{2}^{2}+\omega_{3}^{2} b_{3}^{2}+\omega_{4}^{2} b_{4}^{2}}{\omega_{1}^{2} a_{1}^{2}+\omega_{2}^{2} a_{2}^{2}+\omega_{3}^{2} a_{3}^{2}+\omega_{4}^{2} a_{4}^{2}} .
$$

The relative dissipation power for the system having energy only on the first mode $\left(a_{2}=a_{3}=a_{4}=0\right)$ is 
represented in Fig. 1e. The peak at $a_{1}=0.24$ indicates the point of maximal performance of the NES. If energy on the primary system is either lower or higher than this value, the relative dissipation power abruptly drops or quickly decreases, respectively. The exact position of the peak for any mode of the primary system (assuming that it is the only activated mode) can be explicitly found considering Eqs. (14) and (21), and it is given by the simple formula

$a_{h \text { peak }}=\frac{4 \zeta_{\mathrm{a}} \omega_{1}}{u_{l h} \sqrt{3}}$,

while the maximum relative dissipation power is

$P_{\mathrm{r}, \max }=\frac{\varepsilon u_{l h}^{2} \omega_{h}^{2}}{2 \zeta_{\mathrm{a}} \omega_{1}}$

The simple Eqs. (22) and (23) provide very useful information about the energy level of optimal performance and maximal relative dissipation power of the NES, which is essential for its engineering design.

For all the modes, the SIM assumes the classical "S" shape, whose folds (marked by red dots in the figure) have coordinates modes possessing only very low initial energy. Figure 2 depicts sections of the SIM, representing the trend of $b_{1}, b_{2}, b_{3}$ and $b_{4}$ for variations of $a_{2}$ (strongly activated mode), while $a_{1}, a_{3}$ and $a_{4}$ are small and fixed at 0.01 (very slightly activated modes). $b_{2}$ is practically unchanged with respect to what illustrated in Fig. 1. Regarding $b_{1}, b_{3}$ and $b_{4}$, we notice that they have significant variations for varying $a_{2}$, although $a_{1}, a_{3}$ and $a_{4}$ are kept fixed. Even though they generally have a small amplitude, the SIM sections referred to the slightly activated modes show curves that resemble a frequency resonance, with a pronounced peak. In order to understand the origin of these peaks and predict their position, we consider the equation of the SIM itself. Considering, for instance, Eq. (19), in correspondence of this sort of resonance, we have that $b_{1}$ and $b_{4}$ are very small, so their squares can be neglected. Similarly, $b_{3}^{2} \ll b_{2}^{2}$, which allows us to reduce the equation to

$$
b_{3}^{2} \approx \frac{u_{23}^{2} \omega_{3}^{4} a_{3}^{2}}{\left(\omega_{3}^{2}-\frac{3}{2} b_{2}^{2}\right)^{2}+4 \zeta_{\mathrm{a}}^{2} \omega_{1}^{4}} .
$$

$$
\begin{aligned}
& \left(\sqrt{\frac{8\left(2 \omega_{h}^{2}-\sqrt{\omega_{h}^{4}-12 \omega_{1}^{2} \omega_{h}^{2} \zeta_{\mathrm{a}}^{2}}\right)\left(12 \omega_{1}^{2} \zeta_{\mathrm{a}}^{2}+\omega_{h}^{2}+\sqrt{\omega_{h}^{4}-12 \omega_{1}^{2} \omega_{h}^{2} \zeta_{\mathrm{a}}^{2}}\right)}{81 u_{l h}^{2} \omega_{h}^{2}}}, \frac{2}{3} \sqrt{2 \omega_{h}^{2}-\sqrt{\omega_{h}^{4}-12 \omega_{1}^{2} \omega_{h}^{2} \zeta_{\mathrm{a}}^{2}}}\right) \\
& \left(\sqrt{\frac{8\left(2 \omega_{h}^{2}+\sqrt{\omega_{h}^{4}-12 \omega_{1}^{2} \omega_{h}^{2} \zeta_{\mathrm{a}}^{2}}\right)\left(12 \omega_{1}^{2} \zeta_{\mathrm{a}}^{2}+\omega_{h}^{2}-\sqrt{\omega_{h}^{4}-12 \omega_{1}^{2} \omega_{h}^{2} \zeta_{\mathrm{a}}^{2}}\right)}{81 u_{l h}^{2} \omega_{h}^{2}}}, \frac{2}{3} \sqrt{2 \omega_{h}^{2}+\sqrt{\omega_{h}^{4}-12 \omega_{1}^{2} \omega_{h}^{2} \zeta_{\mathrm{a}}^{2}}}\right) .
\end{aligned}
$$

Comparing Fig. 1a and e, it can be recognized that the NES has optimal performance in the vicinity of the upper fold, for an amplitude of the primary system slightly larger than the fold.

\subsection{Interaction points}

In general, vibration energy is shared by more than one mode of the primary system. We now consider the case of one mode initially strongly activated and other
According to Eq. (25), $b_{3}$ has a maximum for

$b_{2}=\sqrt{\frac{2}{3}} \omega_{3}$

that corresponds to

$$
a_{2} \approx \sqrt{\frac{2}{3}} \frac{\omega_{3}}{u_{22} \omega_{2}^{2}} \sqrt{\left(\omega_{2}^{2}-\frac{\omega_{3}^{2}}{2}\right)^{2}+4 \zeta_{\mathrm{a}}^{2} \omega_{1}^{2} \omega_{2}^{2}} .
$$


(a)

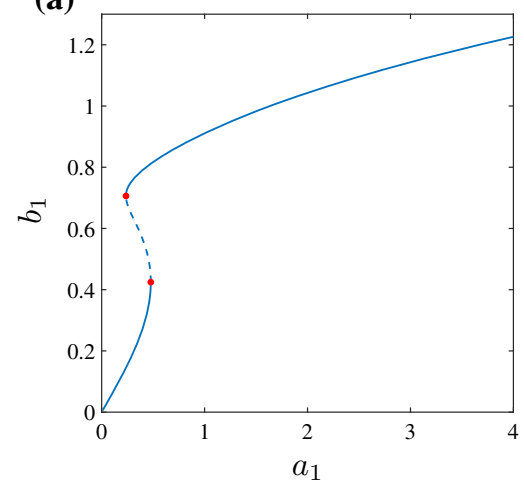

(b)

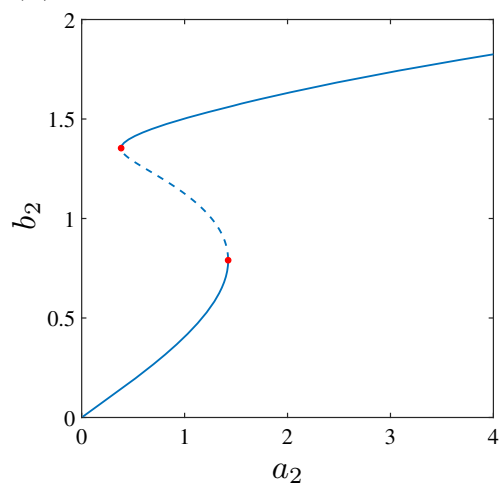

(c)

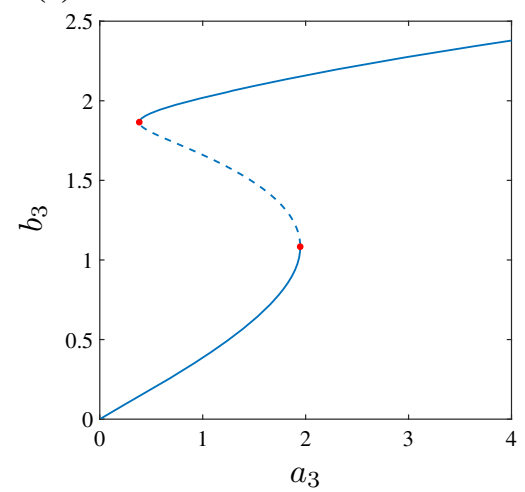

(d)

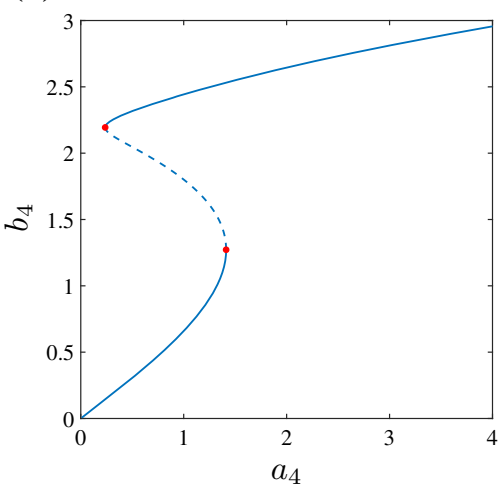

(e)

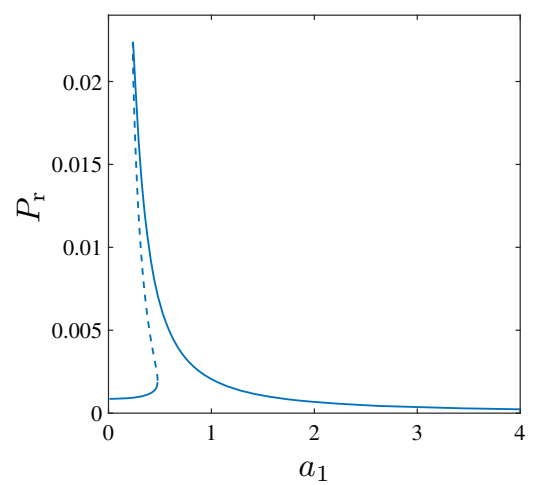

Fig. 1 a-d Sections of the SIM in the $a_{h}, b_{h}$ space when a single mode is activated; e relative dissipation power for the first mode. In each subplot, the not-represented modes are set to zero. Solid lines: stable branches, dashed lines: unstable branches

For the system considered here, these equations provide $a_{2}=0.418$ and $b_{2}=1.321$. The same reasoning can also be done for $b_{1}$ and $b_{4}$, obtaining the values $a_{2}=1.179, b_{2}=0.505$ and $a_{2}=1.364$, $b_{2}=1.553$, respectively. These values, marked by magenta dots in Fig. $2 \mathrm{~b}$, are here named interaction points. The peaks in Fig. 2a, c and d are estimated by $b_{h}=a_{h} u_{2 h} \omega_{h} /\left(2 \zeta_{\mathrm{a}} \omega_{1}\right)$, which, for the system under study, gives $0.030,0.049$ and 0.093 , respectively.

Considering a general $n$-DoF system with attached NES, as in Eq. (7), the coordinates of the interaction point $b_{h}-b_{k}$ can be generalized in the $a_{h}, b_{h}$ space and they are given by

$$
\left(\sqrt{\frac{2}{3}} \frac{\omega_{k}}{u_{l h} \omega_{h}^{2}} \sqrt{\left(\omega_{h}^{2}-\frac{\omega_{k}^{2}}{2}\right)^{2}}+4 \zeta_{\mathrm{a}}^{2} \omega_{1}^{2} \omega_{h}^{2}, \sqrt{\frac{2}{3} \omega_{k}}\right) .
$$

The nomenclature $b_{h}-b_{k}$ indicates the point where energy on the $k$ th mode mainly interacts with the $h^{\text {th }}$ mode; in Fig. $2 \mathrm{~b}$, interaction points $b_{2}-b_{1}, b_{2}-b_{3}$ and $b_{2}$ $b_{4}$ are represented. As it will be also shown in the next subsection, the above-described peaks and the ensuing interaction points are qualitatively significant to explain the modal interaction. In particular, they enable a rapid qualitative prediction of how the presence of a small amount of energy on modes different from the main activated one affects the overall dissipation.

\subsection{Effect of slightly activated modes on a strongly activated mode}

Assuming that most of the energy of the primary system is initially concentrated on the second mode, we analyze the effect of gradually increasing the energy on the other modes. In particular, we start by introducing additional energy on the third mode of the primary sys- 
Fig. 2 Sections of the SIM represented with respect to variations of $a_{2}$ for $a_{1}=a_{3}=a_{4}=0.01$. Magenta dots represent interaction points and are given by Eq. (26). Solid lines: stable branches, dashed lines: unstable branches (a)

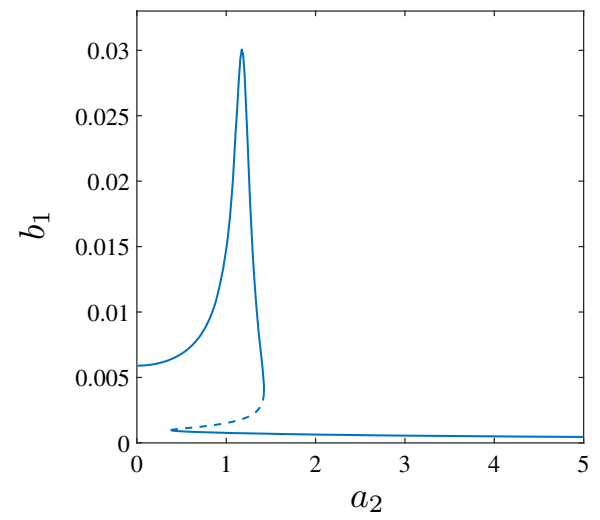

(c)

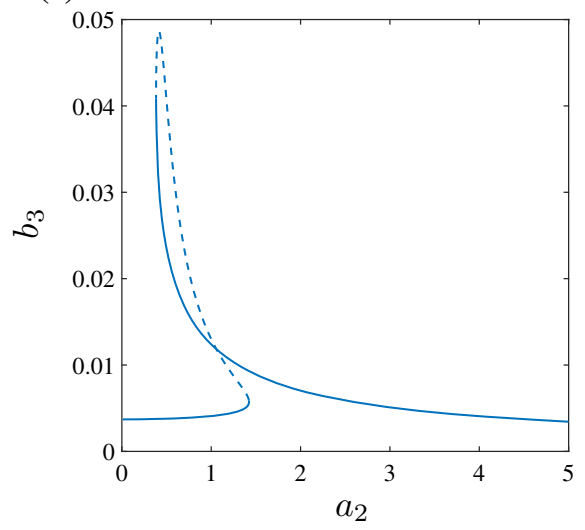

(b)

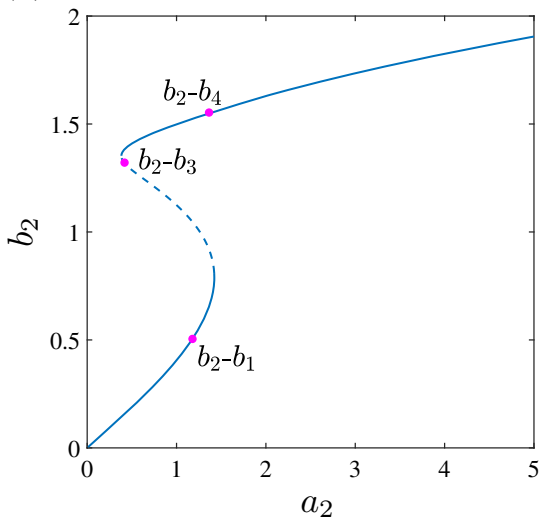

(d)

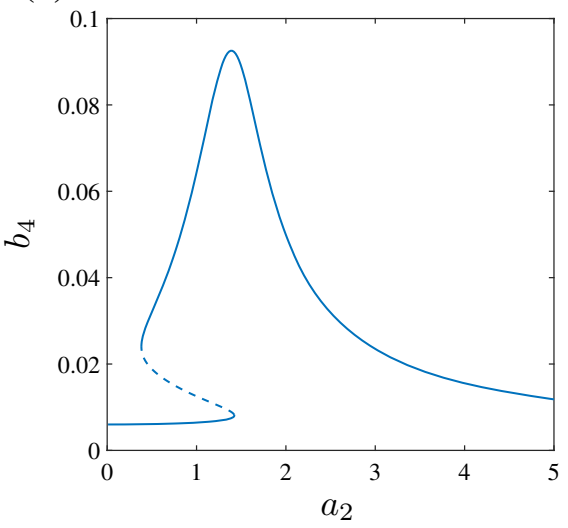

tem. By keeping $a_{1}=a_{4}=0.01$, sections of the SIM for $a_{3}=0.01,0.1,0.2$ and 0.3 are depicted in Fig. 3 . Looking at Fig. 3a, we see that incrementing $a_{3}$ in correspondence of the interaction point (magenta dot) found in the previous section, there is a sort of tongue, which enlarges as $a_{3}$ increases. This is clearly due to the increased contribution of $b_{3}$ in Eq. (18). The peak in $b_{3}$ (Fig. 3b) tends to bend to the right for growing values of $a_{3}$. (Notice that $b_{3}$ is normalized with respect to $a_{3}$ in Fig. 3b.) This is related to the increased relevance of $b_{3}$ with respect to $b_{2}$, which makes the inequality $b_{3} \ll b_{2}$ less accurate. Equation (25) reads now

$$
b_{3}^{2} \approx \frac{u_{23}^{2} \omega_{3}^{2} a_{3}^{2}}{\left(\omega_{3}^{2}+\omega_{a}^{2}-\frac{3}{2} b_{2}^{2}-\frac{3}{4} b_{3}^{2}\right)^{2}+4 \zeta_{\mathrm{a}}^{2} \omega_{1}^{2} \omega_{2}^{2}}
$$

From a practical point of view, this might have an important effect on the dissipation of the second mode. Let us assume that our main objective is to dissipate energy on the second mode. The fact that there is energy on the third mode of the primary system moves the fold to the right, changing the energy level at which the NES works optimally for the second mode. This can be deducted from Fig. 3a by comparing the line corresponding to $a_{3}=0.01$, which shows an upper fold for $a_{2}=0.38$, and the one for $a_{3}=0.3$, which has a fold for $a_{2}=1.36$.

This interpretation is validated by the numerical results shown by the red curves in Fig. $4 a$ and $b$, in which the analytically obtained SIM for $a_{3}=0.3$ (blue curves) is also depicted. In order to keep $a_{3}$ constant during the simulation (necessary to compare analytical and numerical results), the right-hand sides of the first, third and fourth equations of Eq. (16) are set equal to zero. This mathematical artifice implies that although all modes are coupled to the NES, energy is dissipated only on the second mode. Even though the quantitative matching between analytical and numerical results is not excellent, the analytically predicted trend of $b_{2}$ and $b_{3}$ is confirmed. While $a_{2}$ decreases due to energy dissipation, $b_{2}$ suddenly drops while, simultaneously, $b_{3}$ increases. In the wavelet transformation of 
Fig. 3 SIM representation with respect to variations in $a_{2}$ for $a_{1}=a_{4}=0.01$ and $a_{3}=0.01,0.1,0.2$ and 0.3 . Solid lines: stable branches, dashed lines: unstable branches (a)

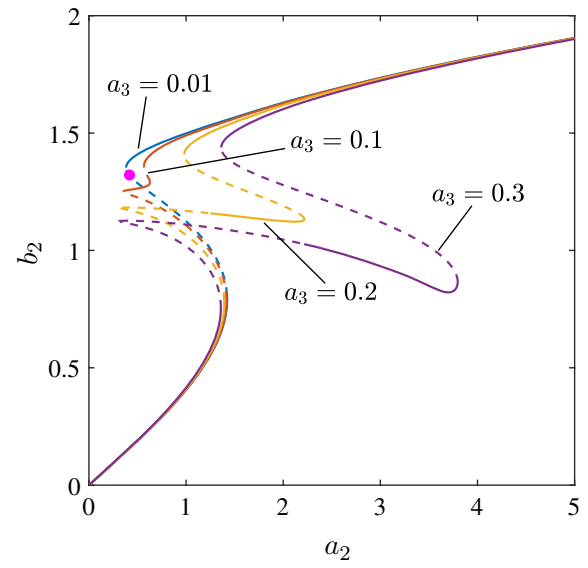

(b)

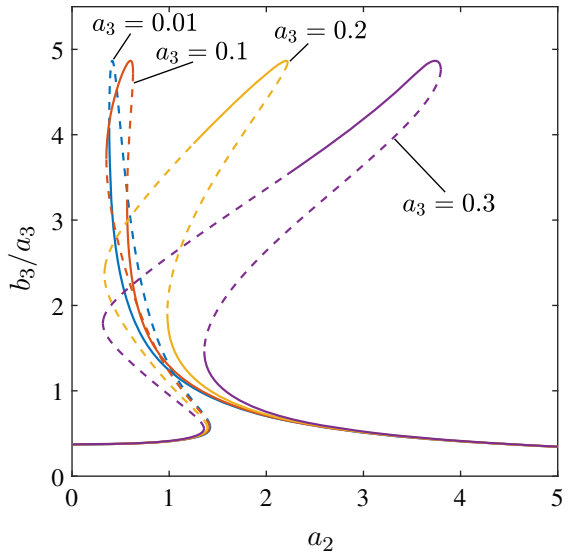

the NES response shown in Fig. 4d, this jump occurring at $T \approx 1900$ is clearly visible. Then, $b_{2}$ slowly increases again and $b_{3}$ decreases, until the NES dynamically disengages from the main structure and both $b_{2}$ and $b_{3}$ jump down to very low values. As it can be recognized from the time series of $y_{2}$ in Fig. $4 c$, the drop of $b_{2}$ significantly influences the energy dissipation of $y_{2}$. The mismatch between numerical and analytical results is most probably related to modal interactions neglected in the present analytical development. In fact, apart from the directly activated frequencies $\omega_{2}=1.18$ and $\omega_{3}=1.62$, the wavelet transformation of Fig. 4d shows the presence of harmonic content at frequencies of $2 \omega_{2}-\omega_{3}=0.73,2 \omega_{3}-\omega_{2}=2.06$ and $3 \omega_{2}-2 \omega_{3}=0.29$. A comparison between the blue and the yellow time series in Fig. 4c clearly illustrates the detrimental effect of the presence of energy on the third mode in terms of energy dissipation.

Stability analysis illustrates that an unstable branch is generated by two Neimark-Sacker bifurcations for $0.32<a_{2}<2.22$ (apart from the unstable branch related to the fold of the SIM for $1.36<a_{2}<$ 3.80). This instability, arguably producing a branch of quasiperiodic solutions, does not seem to have a significant role in the dissipation performance of the NES, since the state of the system in the slow dynamics remains in the vicinity of this unstable branch.

The black dotted lines in Fig. 4a and $4 \mathrm{~b}$ refer to a fictitious numerical simulations obtained by changing the sign of the right-hand side of Eq. (16). This makes the NES artificially pump energy in the system, instead of dissipating it. This simulation, which is merely a mathematical abstraction without physical sense, enables us to verify the correctness of the lower branch of the SIM obtained analytically.

The same analysis can be performed considering a non-negligible value of $a_{4}$. Imposing $a_{4}=0.2$ (keeping $a_{1}=a_{3}=0.01$ ), we obtain sections of the SIM, time series and the wavelet transformation illustrated in Fig. 5. Figures 4 and 5 share exactly the same qualitative features. The tongue in Fig. 5a starts in correspondence of the magenta dot marking the $b_{2}-b_{4}$ interaction, which lies slightly above the one related to the $b_{2}-b_{3}$ interaction (see Fig. 2b for comparison). The wavelet transformation of Fig. 5d illustrates the existence of internal resonances at frequencies of $2 \omega_{2}-\omega_{4}=0.45$ and $2 \omega_{4}-\omega_{2}=2.63$ apart from $\omega_{2}=1.18$ and $\omega_{4}=1.90$. Also for this case, the time series (Fig. 5c) comparing the decrement of $y_{2}$ with $a_{4}=0.2$ (blue line) and $a_{4}=0.01$ (yellow line) clearly show the detrimental effect of $a_{4}$.

We now consider a small non-negligible value of $a_{1}$, which is a mode smaller than the activated one $a_{2}$. Before analyzing the numerical results, we already notice from Fig. $2 b$ that the magenta point corresponding to the $b_{2}-b_{1}$ interaction lies below the fold of the SIM. As it was extensively discussed in the literature $[13,15]$, this part of the manifold corresponds to very poor performance of the NES, since the ratio between the NES relative oscillation amplitude and the primary system oscillation amplitude is low. Imposing $a_{1}=0.5$, we see from Fig. $6 a$ that no tongue is generated, but only a small bump appears, which increases the value of $b_{2}$, having an opposite effect to the one provided by non-negligible $a_{3}$ or $a_{4}$ values. This implies that the effect of $a_{1}$ is not detrimental with respect to 
Fig. 4 a,b Sections of the SIM represented with respect to variations in $a_{2}$ for $a_{1}=a_{4}=0.01$ and $a_{3}=0.3$. Blue lines: analytical SIM, red lines: numerical results obtained by wavelet transformations, black dotted lines: numerical results obtained by wavelet transformations inverting sign of the right-hand side of Eq. (16), dotted blue line: the case of $a_{3}=0.01$; dashed lines: unstable branches; $\mathbf{c}$ time decrement of $y_{2}$, blue line: $a_{3}=0.3$, yellow line: $a_{3}=0.01 ; \mathbf{d}$ wavelet transformation of the absorber displacement, relative to the case of $a_{3}=0.3$ (a)

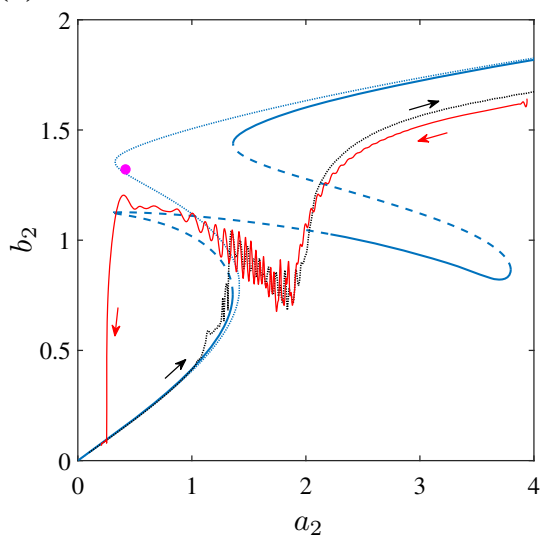

(b)

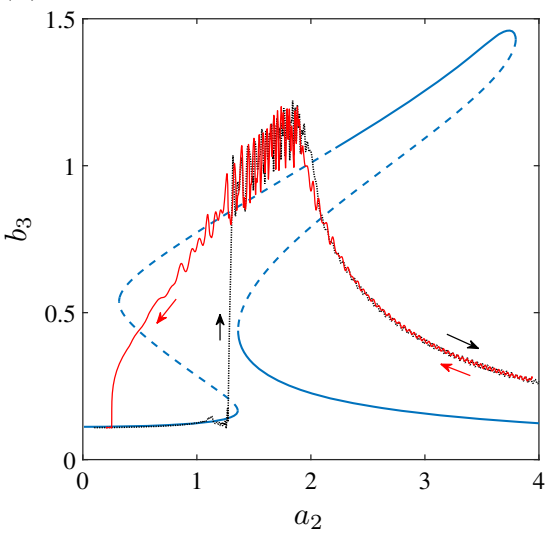

(c)

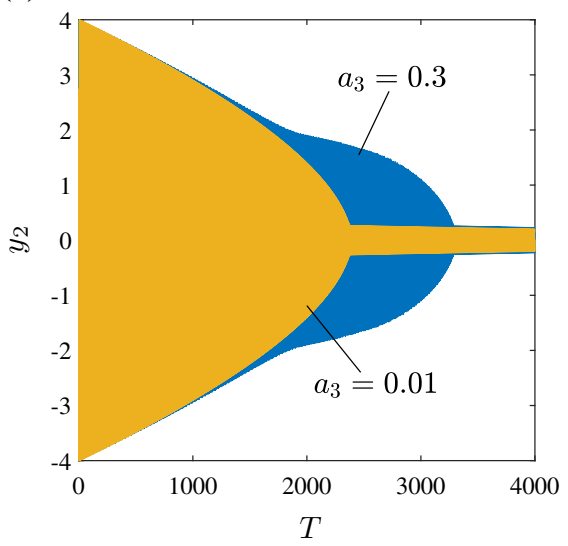

(d)

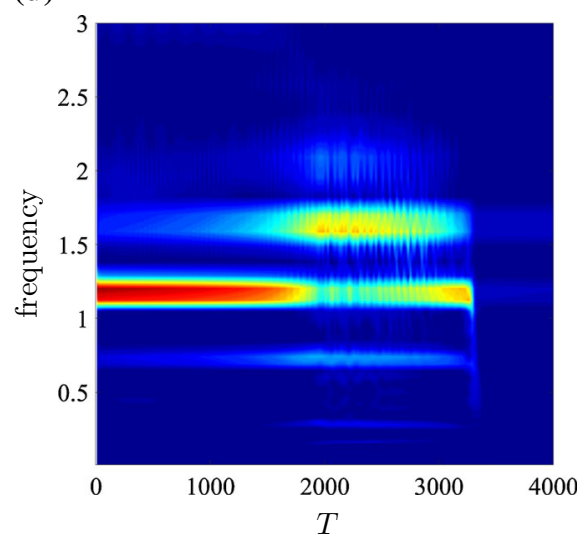

energy dissipation. The red lines in Fig. 6a and b, referring to numerical simulations performed keeping artificially $a_{1}$ constant (right-hand side of the first equation of Eq. (16) is imposed equal to zero), show a good agreement between numerical and analytical results. The jump-down of $b_{2}$ is well predicted, while there is a slight mismatch between analytical and numerical manifolds in the upper branch of $b_{2}$ (lower branch of $b_{1}$ ). The wavelet transformation (Fig. 6d) shows the presence of a modal interaction for the frequency of $2 \omega_{2}-\omega_{1}=1.73$, which is probably causing the mismatch. After the jump-down of $b_{2}$ at $T=1400$, the amplitudes of $b_{1}$ and $b_{2}$ are well predicted. The only additional harmonics identifiable from the wavelet transformation after the jump are $2 \omega_{1}-\omega_{2}=0.06$ and $3 \omega_{1}=1.85$; however, they are very weak (and only hardly detectable from the figure). Figure $6 c$ clearly shows that the energy on the second mode is smoothly dissipated until the fold of the manifold is reached, whose position is not affected by $a_{1}$. Besides, com- paring the case of $a_{1}=0.5$ and $a_{1}=0.01$, it can be recognized that after the jump-down of $b_{2}(T \approx 1450)$, the presence of $a_{1}$ improves energy dissipation on the second mode thanks to the small bump of $b_{2}$ illustrated in Fig. 6a. We remark that, for the case of $a_{1}=0.5$, a couple of Neimark-Sacker bifurcations delimits a large unstable part of the lower branch. The effect of this instability is not disclosed by the present analysis, because for $a_{2}$ values corresponding to the instability, the state of the system in the slow dynamics lies in the vicinity of the upper branch. Nevertheless, acknowledging that the lower branch corresponds to poor performance, we do not expect that this instability is particularly relevant from an engineering point of view.

The analysis so far performed illustrates that nonnegligible values of slightly activated modes can have a significant effect on the energy dissipation of the activated mode. This effect can be partially predicted based on the position of the interaction points. As it was also shown in previous studies $[13,15,16]$, the NES has opti- 
Fig. 5 a,b Sections of the SIM represented with respect to variations in $a_{2}$ for $a_{1}=a_{3}=0.01$ and $a_{4}=0.2$. Blue lines: analytical SIM, red lines: numerical results obtained by wavelet transformations, dashed blue line: the case of $a_{4}=0.01$, dashed lines: unstable branches; $\mathbf{c}$ time decrement of $y_{2}$, blue line: $a_{4}=0.2$, yellow line: $a_{4}=0.01 ; \mathbf{d}$ wavelet transformation of the absorber displacement, relative to the case of $a_{4}=0.2$ (a)

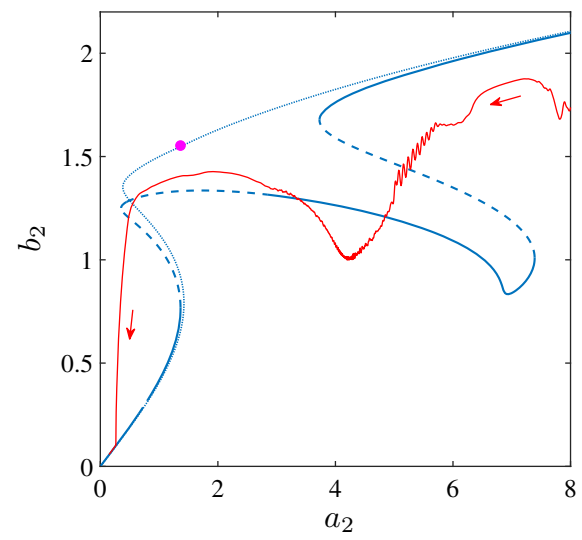

(b)

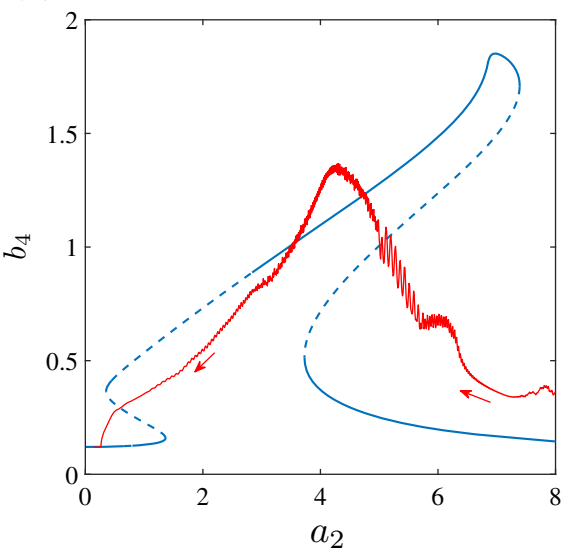

(c)

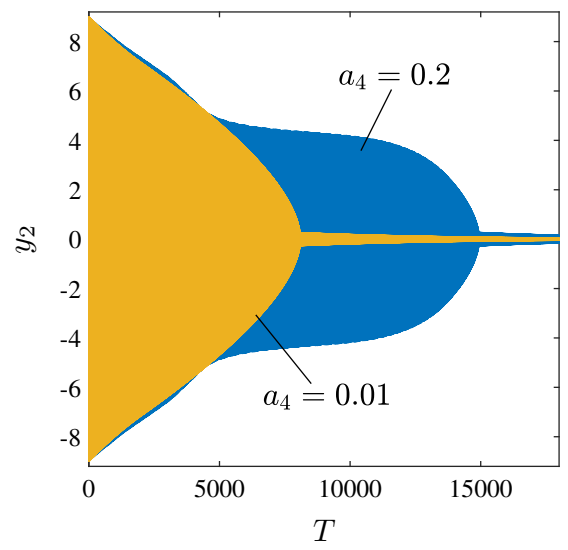

(d)

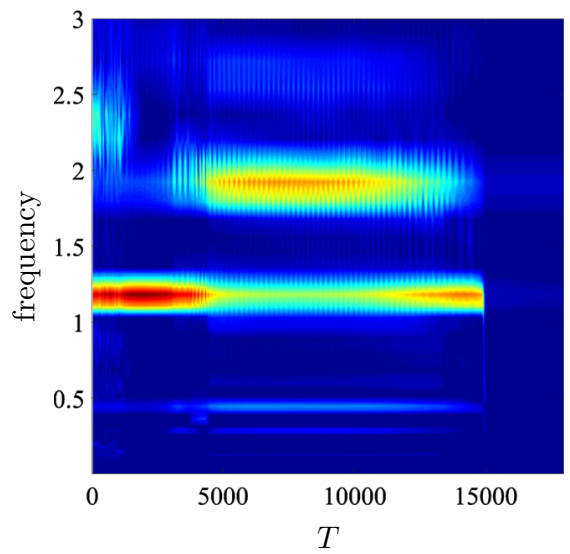

mal dissipation energy in the vicinity of the upper fold of the SIM; therefore, if an interaction point lies near the upper fold, the corresponding mode can have an impact on energy dissipation properties of the other mode. Comparing Eqs. (24) and (26), it can be recognized that resonance points related to lower modes always lie below the upper fold, while resonance points related to upper modes may lie either above or below. The higher is the mode, the higher is the position of the interaction point on the manifold. As a general rule, if the two resonances are close, interaction points might lie in the vicinity of the fold, while if they are very far from each other, they will lie far from the upper fold.

In Fig. 7, this trend can be verified for the first, third and fourth modes of the system under study. From Fig. 7a, it can be inferred that the second mode has a strong impact on the first one, while the third and the fourth ones have effects only at high amplitude, far from optimal behavior of the NES on the first mode.
This is confirmed by the red curve in the figure, where $a_{2}, a_{3}$ and $a_{4}$ are different from zero. Figure $7 \mathrm{~b}$ shows that the first and second interaction points lie on the lower branch, so small non-negligible values of $a_{1}$ and $a_{2}$ would not significantly affect NES dissipation performance on the third mode; $b_{3}-b_{4}$ interaction point, instead, lies in between the two folds of the manifold; it is therefore difficult to ascertain a priori whether small $a_{4}$ values would significantly affect performance of NES or not. However, the red curve in Fig. 7b, depicting the SIM for a small non-negligible values of the $a_{4}$, illustrates that the introduction of energy on the fourth mode modifies the manifold in a way which should not deteriorate energy dissipation on the third mode. In Fig. 7c, referring to the fourth mode, all interaction points lie on the lower SIM branch, suggesting that the NES performance on the fourth mode is rather robust against small values of $a_{1}, a_{2}$ or $a_{3}$. This is also con- 
Fig. 6 a,b Sections of the SIM represented with respect to variations in $a_{2}$ for $a_{3}=a_{4}=0.01$ and $a_{1}=0.5$. Blue lines: analytical SIM, red lines: numerical results obtained by wavelet transformations, dashed blue line: the case of $a_{1}=0.01$, dashed lines: unstable branches; $\mathbf{c}$ time decrement of $y_{2}$, blue line: $a_{1}=0.5$, yellow line: $a_{1}=0.01 ; \mathbf{d}$ wavelet transformation of the absorber displacement, relative to the case of $a_{1}=0.5$
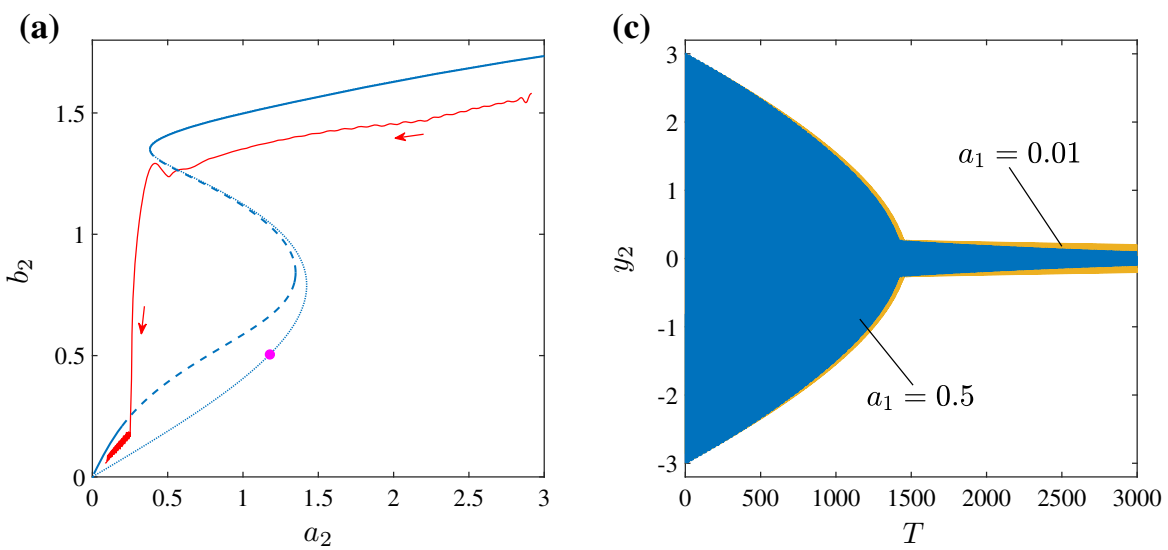

(b)

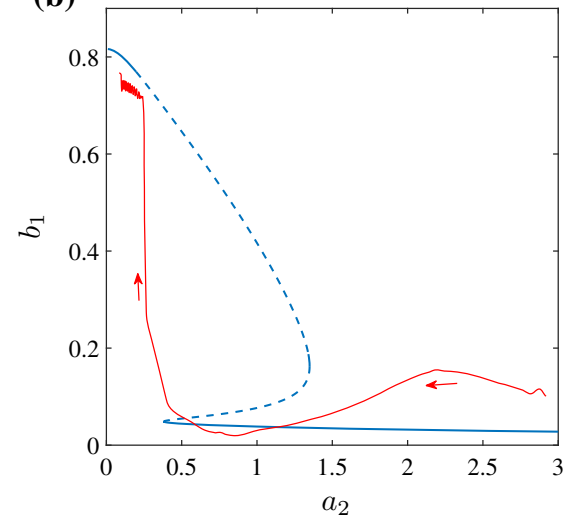

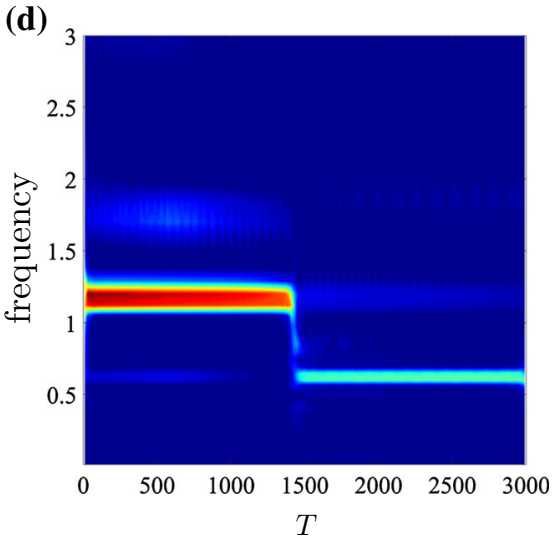

firmed by the red curve in Fig. 7c, which is almost overlapped on the blue one.

We remark that in this section only small values of the non-activated modes were considered. If more than one mode of the primary system possesses a significant amount of energy, the analysis is much more involved, as it will be partially discussed in the next section.

\section{Resonance capture cascade}

Several research works $[1,5,8,13,18]$ already illustrated that an NES attached to a multi-DoF system tends to first dissipate energy on higher modes and then on lower modes, generating the so-called resonance capture cascade (RCC). In the present study, we aim at illustrating and interpreting this phenomenon exploiting the SIM.

At first, we assume that only the first two modes are activated, with initial displacements $y_{1}(0)=$ $y_{2}(0)=3$. Referring to a direct numerical simulation of Eq. (16), Fig. 8a and b depicts the time series of $y_{1}$ and $y_{2}$, Fig. $8 \mathrm{c}$ shows the wavelet transformation of the NES relative displacement $\left(y_{5}\right)$, while Fig. $8 d$ depicts the amplitude of oscillation of the NES relative displacement on the first and second modes (blue and red lines, respectively), obtained from the wavelet transformation. The wavelet transformation clearly illustrates that initially the NES vibrates according to the second mode (with frequency 1.18), while at $T \approx T_{1}=1150$ it partially disengages from the second mode and it oscillates according to the first mode, until, at $T \approx T_{2}=$ 5080, it disengages also from the first one. This kind of behavior is usually referred to as RCC. The energy initially present at $f=1.73$ is related to the modal interaction $2 \omega_{2}-\omega_{1}=1.73$.

Given that both $a_{1}$ and $a_{2}$ vary, the interpretation of the dynamics is carried out through SIM sections. Figure $9 \mathrm{a}-\mathrm{c}$ depicts sections of the SIM in the $a_{1}, b_{1}$, $a_{2}, b_{2}$ and $a_{2}, b_{1}$ spaces, respectively, for various $a_{1}$ and $a_{2}$ values. From Fig. 9a and b, it can be immediately recognized that the value $a_{2}=3$ drastically reduces the initial value of $b_{1}$, while $a_{1}=3$ has prac- 
(a)

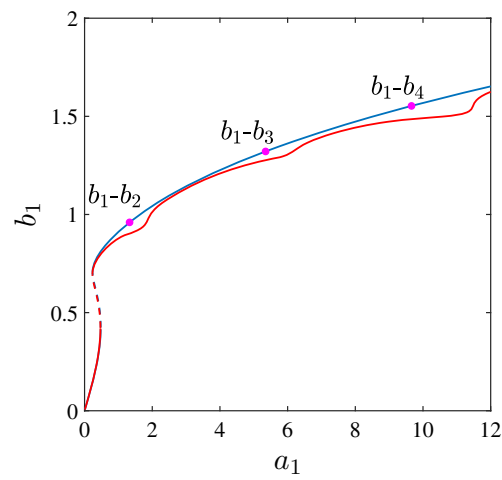

(b)

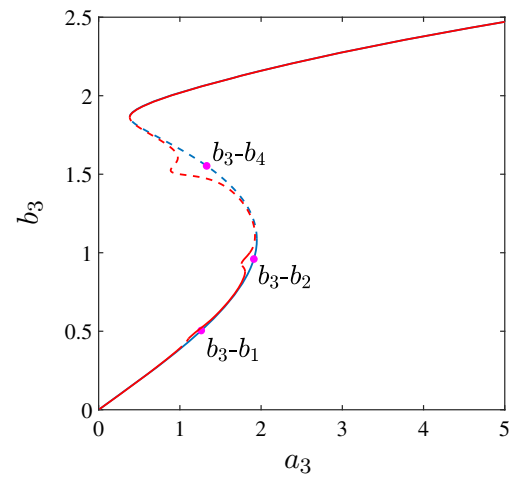

(c)

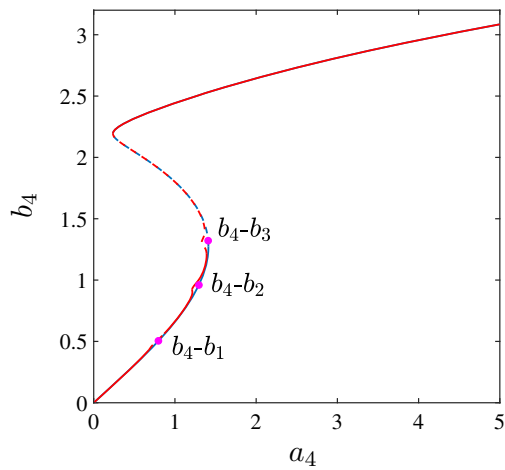

Fig. 7 Sections of the SIM in the $a_{1}, b_{1}$ (a), $a_{3}, b_{3}$ (b) and $a_{4}, b_{4}$ (c) spaces. Blue lines represent the manifold for a single mode activated $\left(a_{2}=a_{3}=a_{4}=0, a_{1}=a_{2}=a_{4}=0\right.$ and $a_{1}=a_{2}=a_{3}=0$, respectively). Red lines mark the man- ifold where the non-represented mode has values $a_{1}=0.1 / \omega_{1}$, $a_{2}=0.1 / \omega_{2}, a_{3}=0.1 / \omega_{3}$ and $a_{4}=0.1 / \omega_{4}$. Magenta dots indicate interaction points with the other modes. Solid lines: stable branches, dashed lines: unstable branches
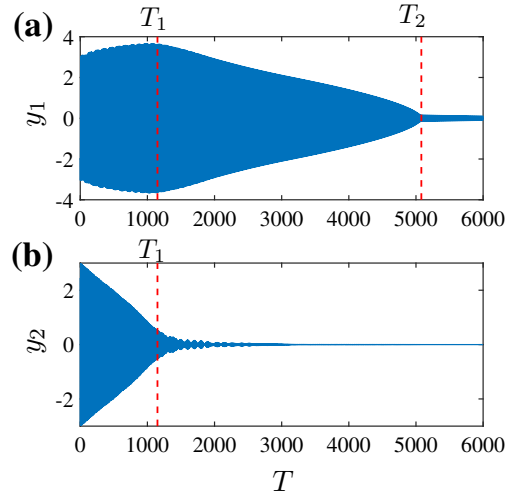

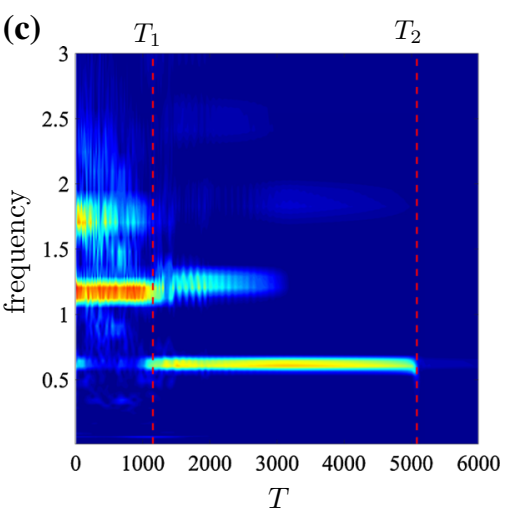

(d)

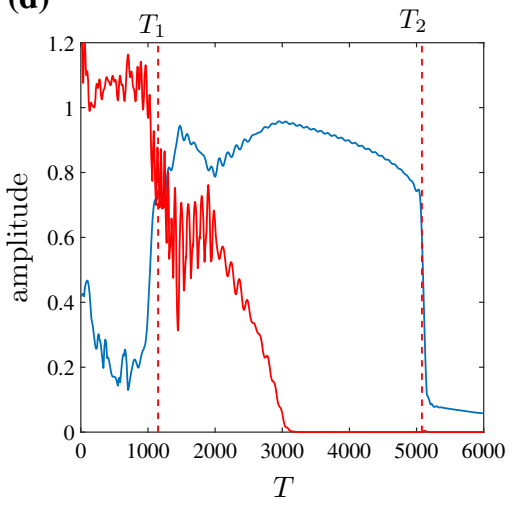

Fig. 8 Numerical simulations of the system in Eq. (16) with initial conditions $y_{1}(0)=y_{2}(0)=3$. a,b Time series; $\mathbf{c}$ wavelet transformation of $y_{5} ; \mathbf{d}$ modal amplitude of oscillation of $y_{5}$ on the first (blue line) and second (red line) modes obtained from the wavelet transformation tically no effect on $b_{2}$ for $a_{2}=3$. Initially, $a_{1}$ and $a_{2}$ are both equal to 3 , which, according to the SIM, corresponds to $b_{1}=0.17$ and $b_{2}=1.7$ (point $T_{0}$ in the figure). Consequently, we expect that energy will be dissipated mainly in the second mode. This observation is consistent with what found numerically, i.e., the NES interacts with the second mode. In order to follow the slow dynamics of the system, we focus on the sections of the SIM in Fig. $9 \mathrm{~b}$ and c, referring to constant $a_{1}=3$. $a_{2}$ decreases up to 0.36 ( $T_{1}$ in the figure), when $b_{2}$ experiences a jump-down and $b_{1}$ a jump-up, which corresponds to the modal shift visible in Fig. 8c at $T_{1}(T \approx 1150)$. At this point, the NES interacts with both modes with a simultaneous decrement of $a_{1}$ and $a_{2}$; therefore, the slow dynamics cannot be directly followed on the sections of the SIM in Fig. 9. However, the rapid decrement of $b_{2}$ illustrated by the wavelet transformation for $1150<T<2670$ can be related to the lower branch of the SIM in Fig. $9 \mathrm{~b}$ for $a_{2}<0.36$. (The lower jump predicted by Fig. 9 is probably smoothed out because $a_{1}$ is not constant). Once most of the energy on the second mode is dissipated, the reference section of the SIM is the orange line in Fig. 9a, which the sys- 
tem follows until $a_{1}=0.24$, corresponding to the NES disengagement occurring at $T_{2}(T \approx 5080)$.

Figure $10 \mathrm{a}$ and $\mathrm{b}$ represents three-dimensional sections of the SIM in the $a_{1}, a_{2}, b_{1}$ and $a_{1}, a_{2}, b_{2}$ spaces, respectively. These plots enable us to visualize the evolution of the slow dynamics obtained numerically on the SIM. It can be recognized that the black lines qualitatively follow the SIM. The quantitative differences are probably related to the presence of additional harmonics, as illustrated in Fig. 8c. The three-dimensional sections of the SIM show that for small energy content in the primary system (small values of $a_{1}$ and $a_{2}$ ), the structure of the manifold is rather involved. On the contrary, for high energy content, the manifold has either high $b_{1}$ and low $b_{2}$ values or the opposite, which means that the NES interacts alternatively with the first or with the second mode. This feature was extensively discussed in [16].

Figure 8a illustrates that, while the NES has 1:1 modal interaction with the second mode $\left(T<T_{1}\right)$, energy not only is not dissipated in the first mode but it also slightly increases. Although this phenomenon cannot be explained with the adopted analytical framework, numerical tests suggest that it is related to the internal resonance which seems to pump energy from the second to the first mode through the NES. This phenomenon occurs also between the third and the second modes and between the fourth and the third modes, as it can be observed in Fig. 11, which represents a RCC involving all four modes of the primary system. Such internal resonances occur at $2 \omega_{n+1}-\omega_{n}$, and the thorough analysis of this phenomenon is out of the scope of this paper.

We now consider the system in Eq. (16) with all the modes initially equally activated. Namely, we impose initial conditions $y_{1}(0)=y_{2}(0)=y_{3}(0)=y_{4}(0)=3$. Time series of the numerical simulations are depicted in Fig. 11a-d, while Fig. 11e illustrates the wavelet transformation of the NES relative displacement $\left(y_{5}\right)$. The time series clearly show the RCC. Initially, the NES resonates with the fourth mode $\left(\omega_{4}=1.90\right)$, which is the only one dissipated for $T<310$. At $T \approx 310\left(T_{1}\right)$, the NES disengages from the fourth mode and starts resonating according to the third mode $\left(\omega_{3}=1.62\right)$ and energy on the third mode is rapidly dissipated while it slightly increases on the second mode. At $T \approx 1030$ $\left(T_{2}\right)$, the NES disengages from the third mode and switches to the second mode and energy on the second mode is now dissipated, while energy slightly increases on the first mode. At $T \approx 2330\left(T_{3}\right)$, the NES finally starts resonating with the first mode and dissipates most of the remaining energy in the system. At $T \approx 6670$ $\left(T_{4}\right)$, the NES disengages also from the first mode and it becomes practically unable to dissipate the remaining energy. The scenario just described represents a typical RCC, and the wavelet transformation of Fig. 11e perfectly visualizes the phenomenon.

The analysis of the RCC through the SIM would imply to consider an eight-dimensional space in which most of the dimensions are relevant for the cascade description. In order to simplify the representation, we assume that the cascade makes the NES dissipate energy exactly from one mode at a time and we try to follow the trend of the slow dynamics through a series of two-dimensional sections of the SIM.

Sections of the SIM for initial conditions $a_{1}=a_{2}=$ $a_{3}=a_{4}=3$ are depicted by the blue lines in Fig. 12 . Comparing the blue with the dotted black lines in each subplot of Fig. 12, we immediately recognized that the amplitudes of $b_{1}, b_{2}$ and $b_{3}$ are strongly reduced by the presence of the other modes, while $b_{4}$ (Fig. 12a) is practically unaffected. This suggests that the NES initially dissipates energy mostly in the fourth mode, as confirmed by the shown numerical simulation.

In order to capture the first jump-down of the cascade from the fourth to the third mode, we focus on the blue lines of Figs. 12a and 13a, which illustrate the variations in $b_{4}$ and $b_{3}$ with respect to $a_{4}$, respectively, for fixed values of $a_{1}, a_{2}$ and $a_{3}$ (whose variations are minimal, as proven numerically). $a_{4}$ decreases due to the high value of $b_{4}$. When $a_{4}$ reaches approximately 0.33 , the two sections of the SIM present a fold (marked by $T_{1}$ in the figures) which makes $b_{4}$ jump down (Fig. 12a), while $b_{3}$ jumps up (Fig. 13a). This point marks the first step of the RCC.

At this stage, the fourth mode is almost completely dissipated; therefore, we approximate $a_{4}$ to 0 , while $a_{1}$, $a_{2}$ and $a_{3}$ are still around 3. Corresponding sections of the SIMs are represented by the orange lines in Fig. 12b, $\mathrm{c}$ and d. According to these sections of the SIM, $b_{3}$ is relatively large, while $b_{1}$ and $b_{2}$ are small, which means that the NES resonates with the third mode. The second jump of the resonance cascade, marked by $T_{2}$ in the figures, can be captured considering the orange curves in Figs. $12 \mathrm{~b}$ and $13 \mathrm{~b}$. When $a_{3}$ reaches $0.36, b_{3}$ experiences a jump-down and $b_{2}$ a jump-up, which marks the second step of the RCC. 
(a)

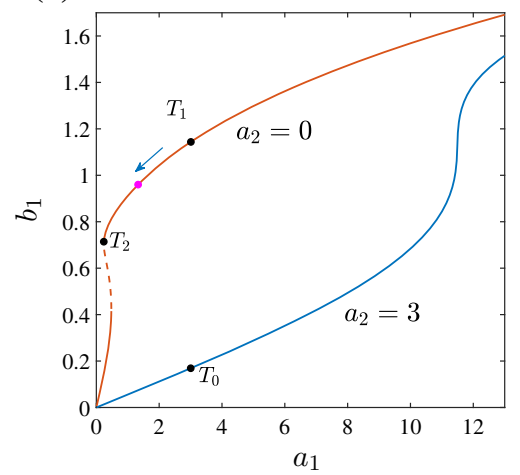

(b)

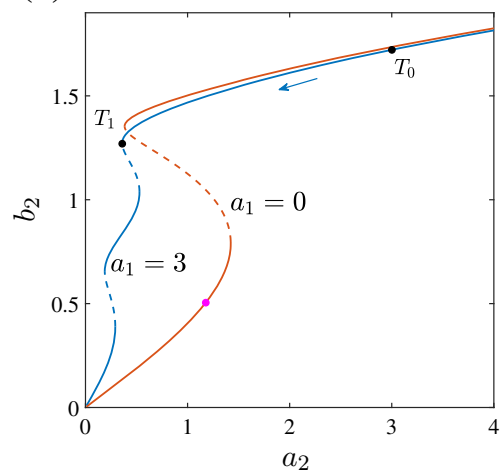

(c)

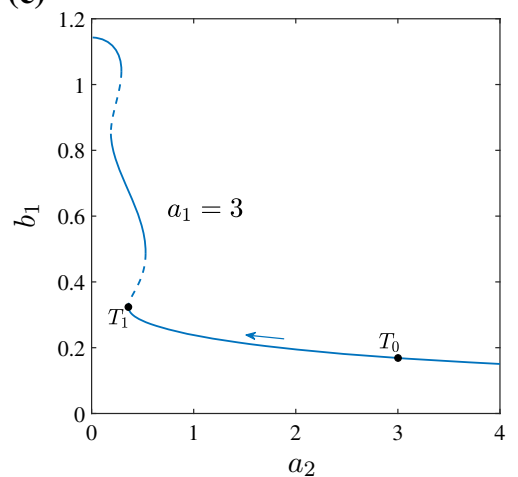

Fig. 9 Sections of the SIM in the $a_{1}, b_{1}$ (a), $a_{2}, b_{2}$ (b) and $a_{2}, b_{1}$ (c) spaces for $a_{3}=a_{4}=0.01$; the values of $a_{1}$ and $a_{2}$ are indicated in the figures. Solid lines: stable branches, dashed lines: unstable branches

Fig. 10 Three-dimensional sections of the SIM for $a_{3}=a_{4}=0.01$ in the $a_{1}, a_{2}, b_{1}$ (a) and $a_{1}, a_{2}, b_{2}$ spaces (b); the thick black lines indicate the path followed by the numerical simulation

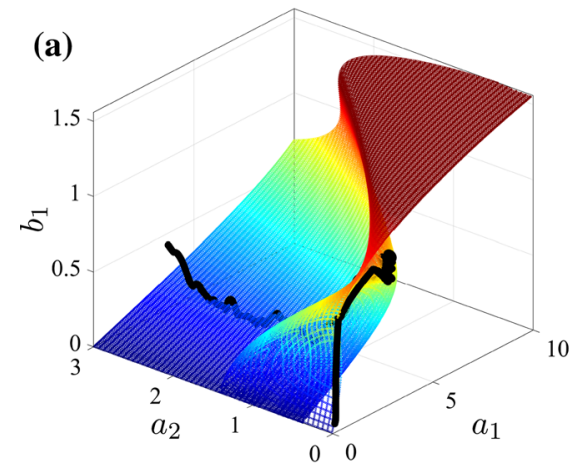

(c) $T_{1} T_{2}$

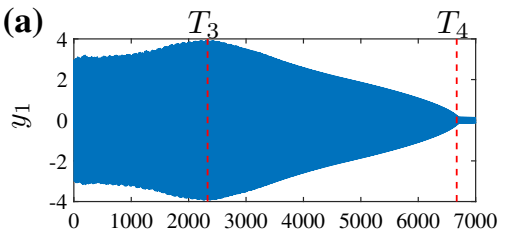

(b)

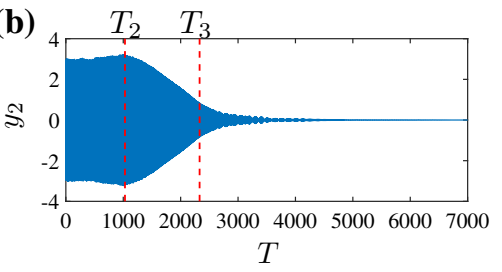

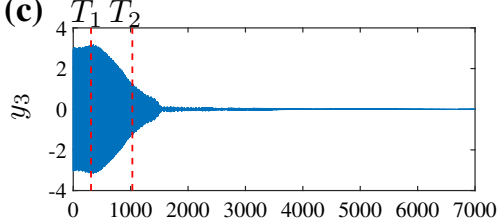

(d)

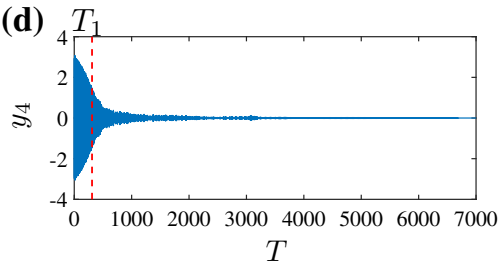

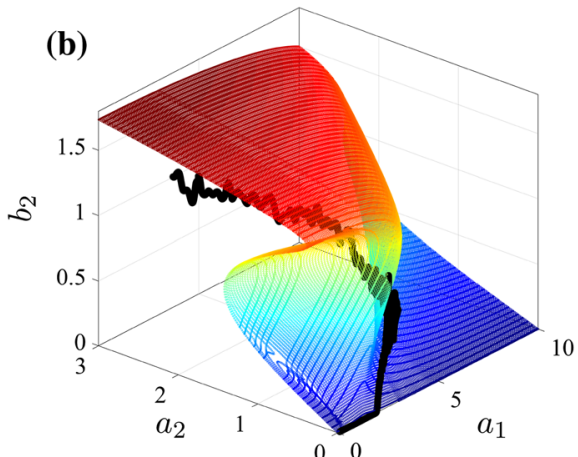

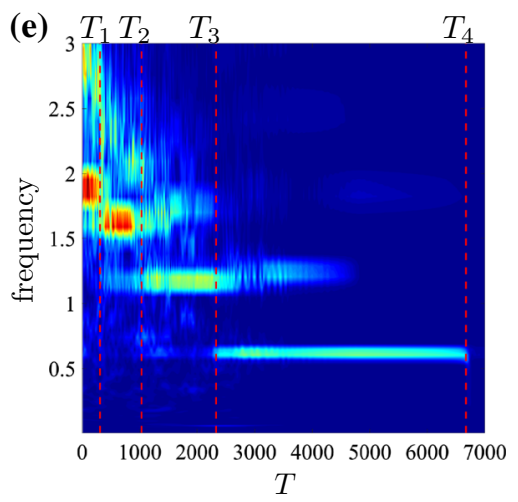

Fig. 11 Numerical simulations of the system in Eq. (16) with initial conditions $y_{1}(0)=y_{2}(0)=y_{3}(0)=y_{4}(0)=3$. a-d Time series; e wavelet transformation of $y_{5}$; dashed red lines indicate frequency jumps 
Fig. 12 Sections of the SIM in the $a_{4}, b_{4}$ (a), $a_{3}, b_{3}$ (b) $a_{2}, b_{2}$ (c) and $a_{1}, b_{1}$ (d) spaces; the other values are indicated in the figures. Solid lines: stable branches, dashed lines: unstable branches. Sections referring to a single activated mode are depicted by dotted black

lines

(a)

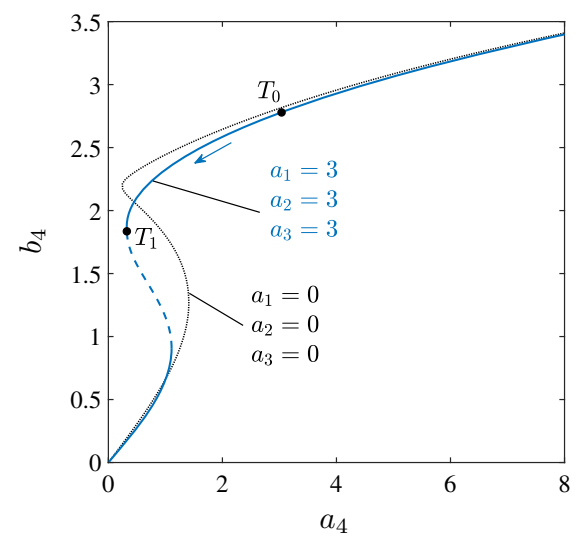

(c)

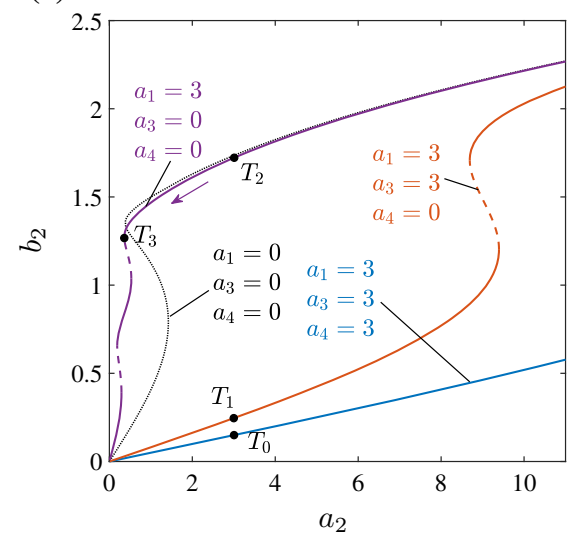

(b)

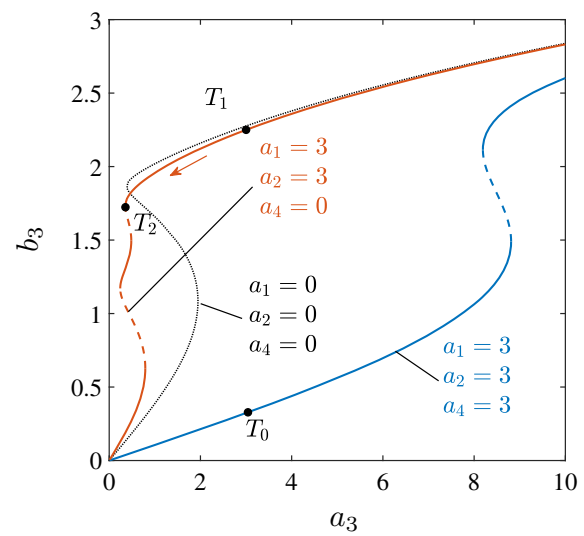

(d)

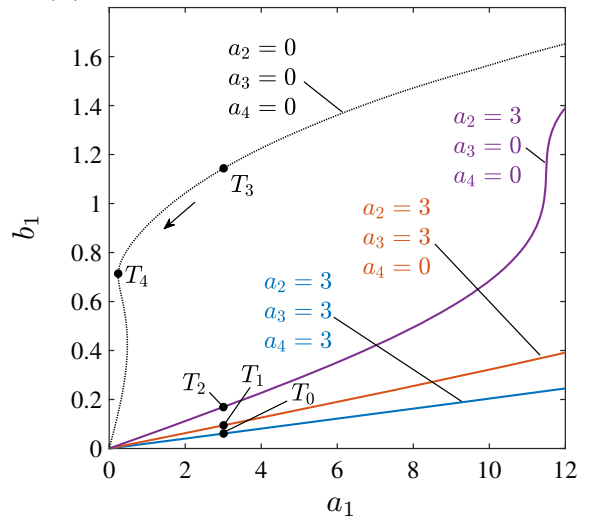

(c)

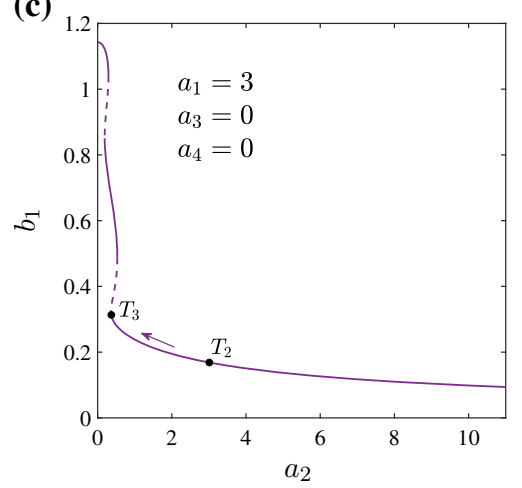

(a)

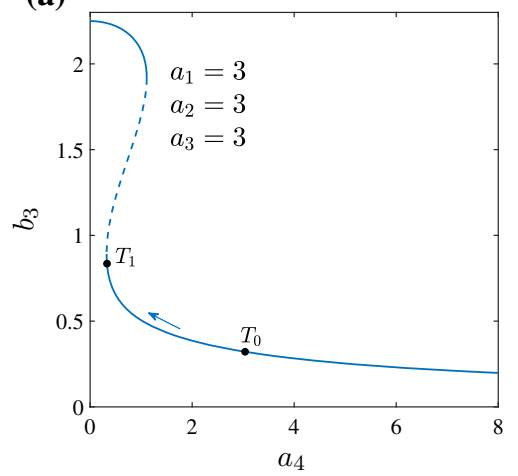

(b)

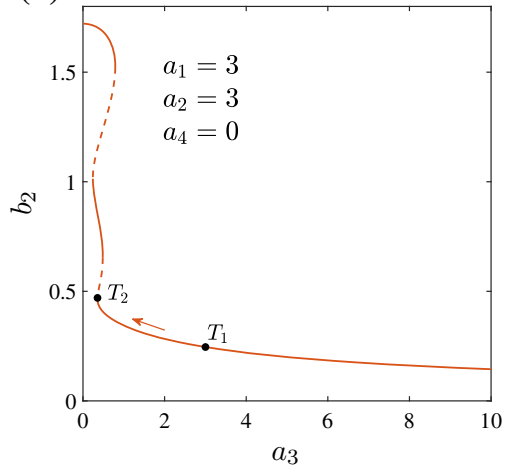

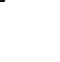


Following the same procedure, we try to visualize the following step of the cascade, i.e., from the second to the first mode. Now, both $a_{3}$ and $a_{4}$ are assumed null, while $a_{1}$ and $a_{2}$ are approximately 3 . Indeed, this is the initial condition considered in the previous numerical simulation (Fig. 8). Corresponding sections of the SIM are given by the purple curves in Fig. 12c and d. These illustrate that $b_{2}$ is much larger than $b_{1}$; therefore, the NES resonates according to the second mode, reducing $a_{2}$ and letting $a_{1}$ almost constant. For $a_{2} \approx 0.36\left(T_{3}\right)$, $b_{2}$ has a jump-down, while $b_{1}$ has a jump-up, marking the third step of the cascade.

Finally, the primary system has energy almost only on the first vibration mode; therefore, the SIM is reduced to the simple case of a single activated mode, depicted by the black dashed line in Fig. 12d. From this moment on, the NES dissipates energy on the first mode, until the fold of the manifold is reached for $a_{1} \approx 0.24\left(T_{4}\right)$, which marks the last step of the RCC.

\section{Performance analysis}

The RCC is an interesting phenomenon characterizing nonlinear vibration absorbers, which, as known, cannot occur with a TMD. In this section, we aim at analyzing its efficiency with respect to the speed of energy dissipation of the NES. Figure 14a depicts the time required to dissipate $80 \%$ of initial energy if the system in Eq. (16) has energy initially equally distributed on all four modes of the primary system. This is obtained by setting initial displacement at zero and modal initial velocity equal for all modes (marked by $\dot{y}_{h}(0)$ in the figure). For very small initial energy, the dissipation time is relatively large, starting from approximately 3200 time units. It then rapidly decreases reaching its minimum for $\dot{y}_{h}(0) \approx 0.65$ (i.e., optimal dissipation time, approximately 400 time units). By further increasing initial energy, the dissipation time increases again in a monotonous way on average, reaching 3150 time units for $\dot{y}_{h}(0)=3.5$, which is almost eight times the optimal one. According to these observations, the considered amplitude range can be roughly divided in three regions. For $\dot{y}_{h}(0)<0.5$, the system is practically below the minimum energy threshold for activating resonances with the NES; however, this limit is not the same for all modes; therefore, dissipation time does not have a discontinuity at $\dot{y}_{h}(0)=0.5$, but it has only a steep decrement. As it is illustrated below, for $0.5<\dot{y}_{h}(0)<1$, apart from the absolute minimum at $\dot{y}_{h}(0) \approx 0.65$, the NES has indicatively optimal performance and it is able to interact with more than one mode at the same time; on the contrary, for $\dot{y}_{h}(0)>1$, the RCC takes place.

In order to investigate whether the RCC occurs for specific initial conditions and if there is a relation between RCC and performance, we define the parameter

$C_{h k}=\frac{E_{k}\left(T_{h}\right)}{E_{k}(0)}$

where $T_{h}$ is the dissipation time of $80 \%$ of the energy of the $h$ th mode and $E_{k}(0)$ and $E_{k}\left(T_{h}\right)$ are the initial energy on the $k$ th mode and its energy at time instant $T_{h}$, respectively. In essence when, for instance, $C_{43}$ is approximately 1, it means that the RCC from the fourth to the third is verified since when $80 \%$ of the energy on the fourth mode is dissipated (marked by time $T=T_{4}$ ), the one on the third mode is practically unchanged $\left(E_{3}\left(T_{4}\right) \approx E_{3}(0)\right)$. Conversely, if $C_{43}$ is significantly smaller than 1, it implies that, when $80 \%$ of the energy on the fourth mode is dissipated, the NES already dissipated energy on the third mode as well $\left(E_{3}\left(T_{4}\right) \ll E_{3}(0)\right)$; therefore, we can state that the RCC between the two modes is not taking place.

Figure 14b-d illustrates the values of $C_{43}, C_{32}$ and $C_{21}$ for the same initial conditions of Fig. 14a. The RCC from the fourth to the third mode and that from the third to the second mode occur for $\dot{y}_{h}(0)>1$; however, the values of $C_{43}$ and $C_{32}$ are rather fuzzy and irregular; it is therefore unclear from which exact value the RCC occurs; the RCC from the second to the first mode occurs approximately for $\dot{y}_{h}(0)>0.65$. In general, for high energy level, the RCC seems to occur always; however, by comparing the trends of $C_{43}, C_{32}$ and $C_{21}$ with the dissipation time in Fig. $14 \mathrm{a}$, it can be recognized that for the energy level of fastest energy dissipation $\left(0.5<\dot{y}_{h}(0)<1\right)$, the RCC does not occur.

Figures 15 and 16 illustrate time series, energy decrement and wavelet transformations for two numerical simulations relative to the initial condition $\dot{y}_{1}(0)=$ $\dot{y}_{2}(0)=\dot{y}_{3}(0)=\dot{y}_{4}(0)=1.3$ and 0.65 , respectively. The former simulation corresponds to a case of relatively fast energy dissipation (dissipation time is 841 time units), which presents a clear RCC, while the latter simulation corresponds to the case of almost fastest 
Fig. 14 Results referred to simulations having initial conditions $y_{1}(0)=y_{2}(0)=$ $y_{3}(0)=y_{4}(0)=0$ and $\dot{y}_{1}(0)=\dot{y}_{2}(0)=\dot{y}_{3}(0)=$ $\dot{y}_{4}(0)=\dot{y}_{h}(0)$ indicated on the $x$-axis of the figures. a Time required to dissipate $80 \%$ of initial energy; $\mathbf{b}-\mathbf{d}$ cascade coefficients $C_{43}$, $C_{32}$ and $C_{21}$ (a)

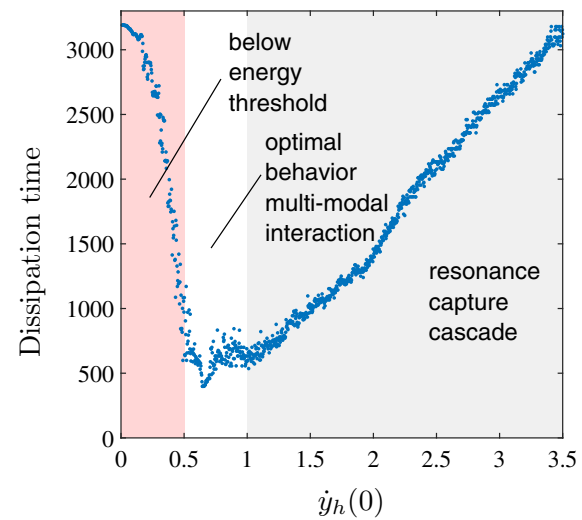

(c)

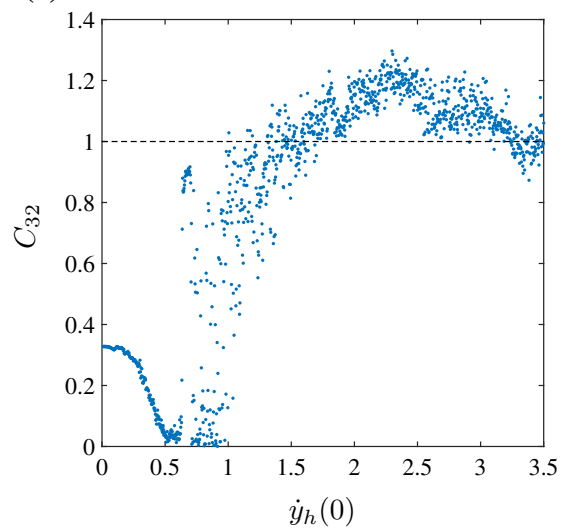

(b)

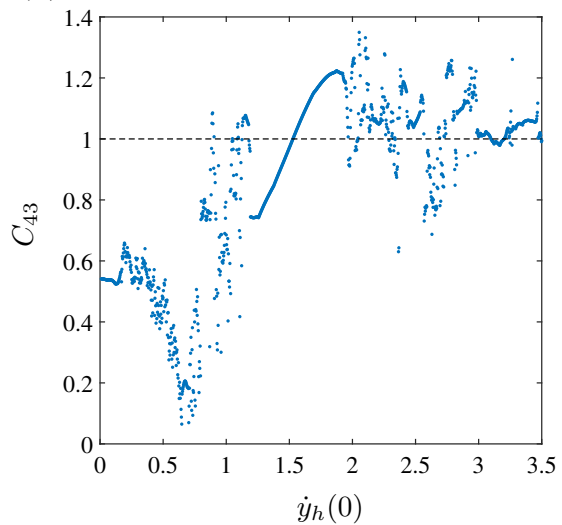

(d)

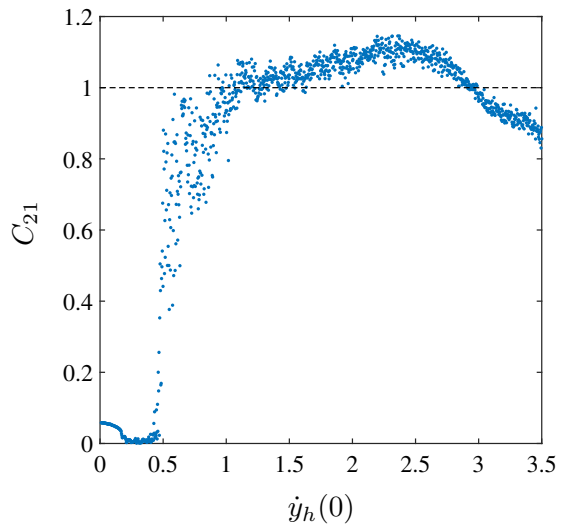

energy dissipation (dissipation time is 409 time units) which only partially presents RCC.

In the first case (Fig. 15), the RCC takes place, as it can be clearly recognized from the energy decrement of Fig. 15b. Energy is first dissipated on the fourth mode, then on the third, successively on the second and finally on the first one (in a relatively long time). Although the numerous subresonances do not facilitate to recognize the steps of the RCC, the wavelet transformation of the NES relative displacement exhibits a clear trend ascribable to the RCC.

In the second case (Fig. 16), the NES dissipates very rapidly energy on the third and fourth modes, practically at the same time. The dissipation of energy in the second mode is slightly slower, while the dissipation of energy in the first mode starts at $T \approx 150$. Overall the dissipation time is 409 time units. Although energy on the third, second and first modes is dissipated resembling a RCC, the NES resonates with the third and fourth modes at the same time, as clearly visible from the wavelet transformation in Fig. 16c. Despite the faster energy dissipation, we notice that in the case in Fig. 16 the residual energy in the second, third and fourth modes is much larger than in the case illustrated in Fig. 15, where RCC occurs.

It is particularly difficult to relate the NES dynamics illustrated in Fig. 16 to the SIM, because energy is dissipated on several modes at the same time. Therefore, in order to follow the dynamics on the SIM, it would be necessary to use all its eight dimensions, making its representation impossible.

However, through several sections of the SIM, it is still possible to shed some light on the dynamical behavior exhibited by the NES in the simulation displayed in Fig. 16. In Fig. 17a-d, sections of the SIM assuming that all four modes have the same velocity $\left(\omega_{1} a_{1}=\omega_{2} a_{2}=\omega_{3} a_{3}=\omega_{4} a_{4}\right)$ are represented. We notice that all the four sections present four bumps. In the $b_{1}$ space (Fig. 17a), the first bump is the highest, in the $b_{2}$ space the second one is the highest, and similarly, in the $b_{3}$ and $b_{4}$ spaces the third and the fourth bumps are the highest, respectively. Considering Fig. 16c, we 
(a)
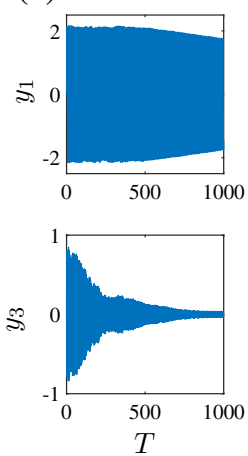

(b)
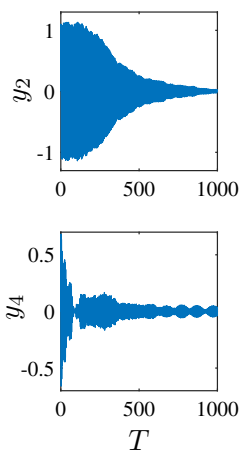

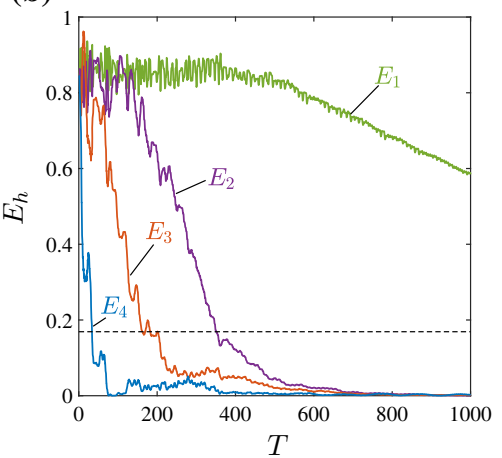

(c)

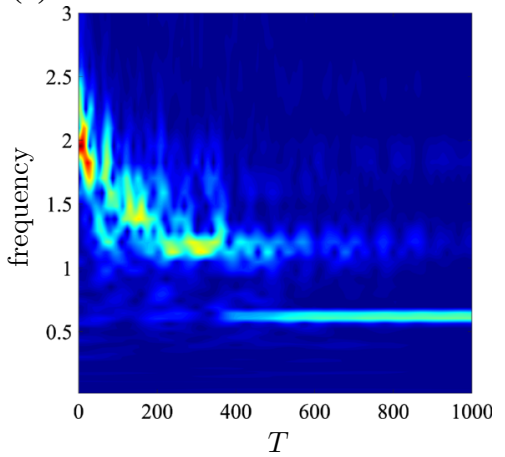

Fig. 15 Numerical simulations of the system in Eq. (16) with initial conditions $\dot{y}_{1}(0)=\dot{y}_{2}(0)=\dot{y}_{3}(0)=\dot{y}_{4}(0)=1.3$. a Time series; b energy decrement for each mode; dashed black line indicates $20 \%$ of initial energy; c wavelet transformation of $y_{5}$

(a)
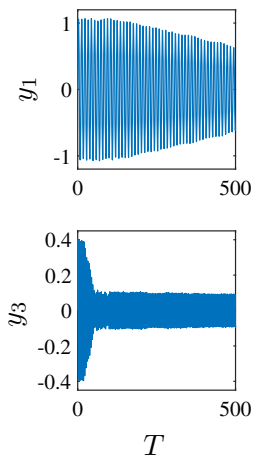
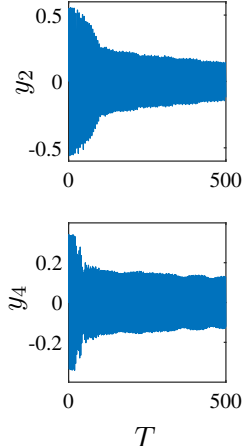

(b)

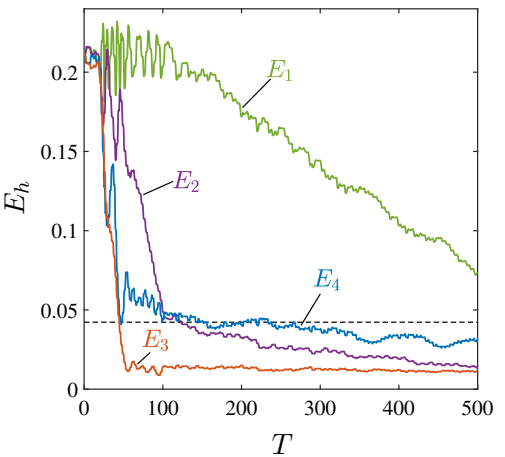

(c)

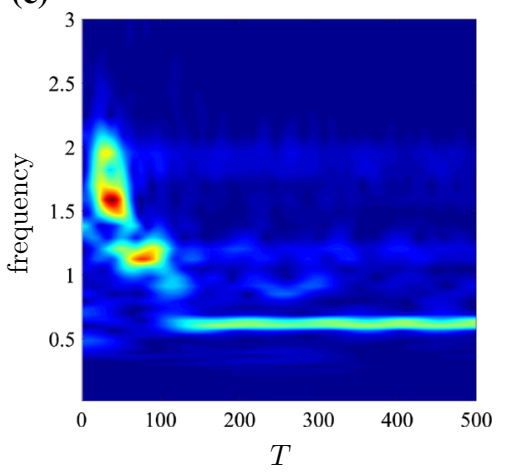

Fig. 16 Numerical simulations of the system in Eq. (16) with initial conditions $\dot{y}_{1}(0)=\dot{y}_{2}(0)=\dot{y}_{3}(0)=\dot{y}_{4}(0)=0.65$. a Time series; b energy decrement for each mode; dashed black line indicates $20 \%$ of initial energy; c wavelet transformation of $y_{5}$

notice that initially the NES has the highest energy on the third mode $\left(\omega_{3}=1.62\right)$, with a simultaneous substantial amount of energy on the fourth mode $\left(\omega_{4}=\right.$ $1.90)$ as well, while initially energy on the second and first modes is very low. Such an energy distribution corresponds to the third bump of Fig. 17a-d, which, for $\omega_{h} a_{h}=0.65$, has $b_{1}=0.068, b_{2}=0.11, b_{3}=1.17$ and $b_{4}=1.11$ (marked by blue dots in Fig. 17a-d). This clearly enables the NES to dissipate energy on the third and fourth modes at the same time, as verified numerically and illustrated in Fig. 16b. Indeed, this is probably the main reason why the NES is able, in this region, to interact with more than one mode at the time, decreasing dissipation time. Successively, the numerical simulation shows that the NES dissipates energy on the second and finally on the first mode; however, these further steps of the energy dissipation cannot be dis- cussed with the SIM sections illustrated, since it is not verified any longer that $\omega_{1} a_{1} \approx \omega_{2} a_{2} \approx \omega_{3} a_{3} \approx \omega_{4} a_{4}$.

SIM sections in Fig. 17a-d enable us to partially explain also the dynamics exhibited in Fig. 15. In this case, we have initially $\omega_{h} a_{h}=1.3$ and energy is first dissipated only on the fourth mode. Such a behavior is probably related to the system lying on the fourth bump, where $b_{1}, b_{2}$ and $b_{3}$ are very small $\left(b_{1}=0.061\right.$, $b_{2}=0.081$ and $b_{3}=0.14$, orange dots in Fig. 17a-c), while $b_{4}$ is rather large $\left(b_{4}=2.36\right)$. After dissipating energy on the fourth mode, the system seemingly follows a path similar to the one described by the SIM sections of Figs. 12 and 13. However, for the sake of brevity, the SIM sections corresponding to this case are omitted here. The relative energy decrement in the two analyzed cases is compared in Fig. 17f.

In Fig. 17e, the relative dissipation power is represented assuming once again that all four modes have the 
(a)

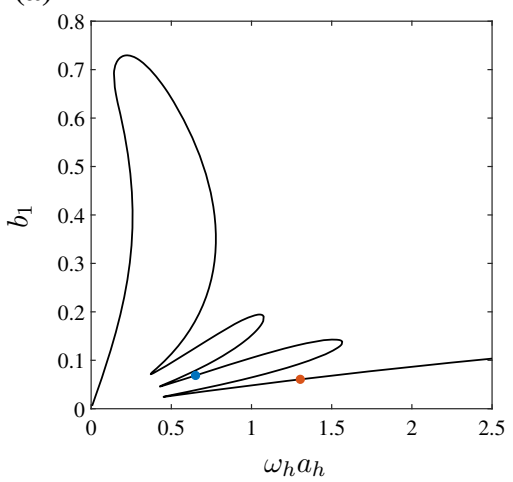

(b)

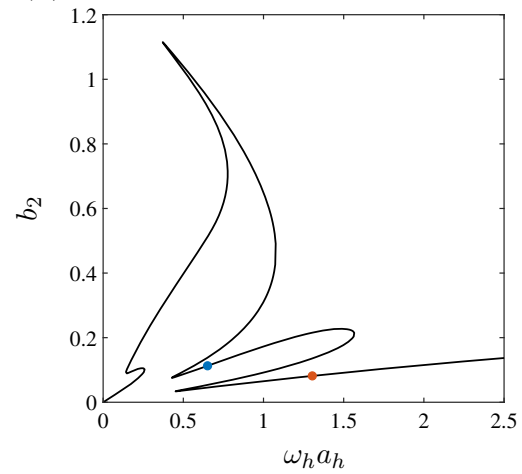

(c)

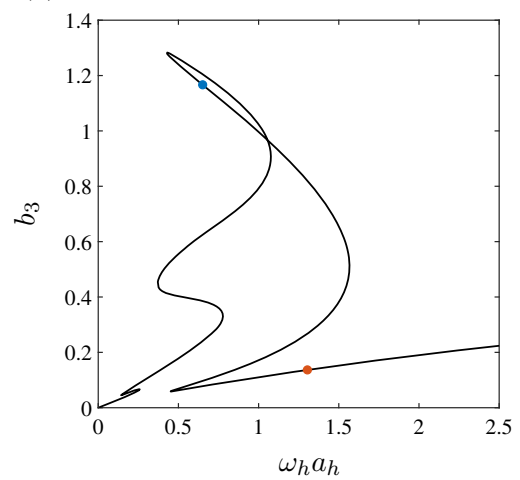

(d)

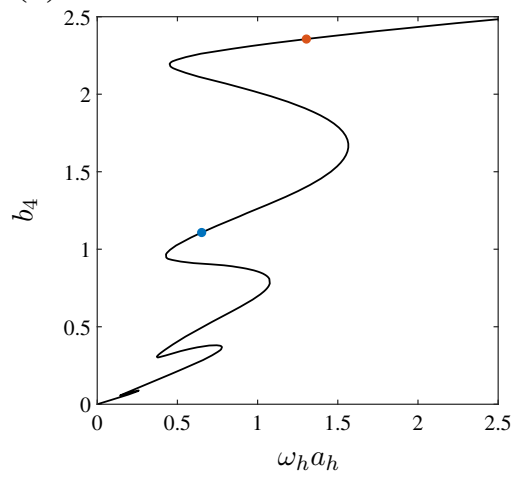

(e)

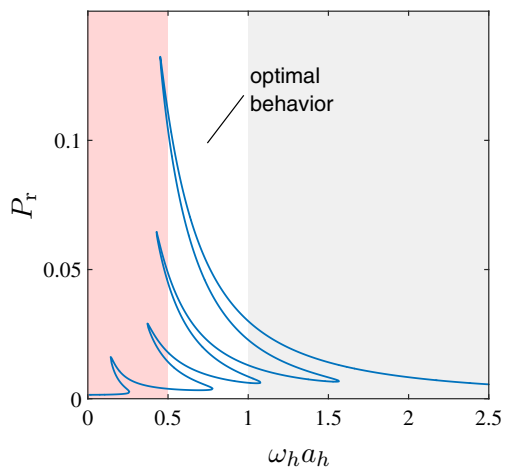

(f)

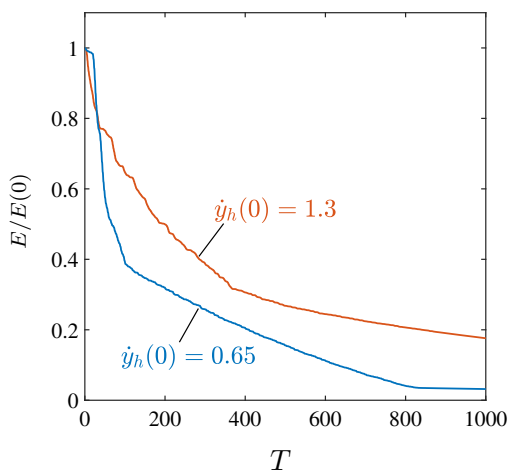

Fig. 17 a-d Sections of the SIM for equal energy distribution on the modes of the primary system $\left(\omega_{1} a_{1}=\omega_{2} a_{2}=\omega_{3} a_{3}=\right.$ $\left.\omega_{4} a_{4}\right)$; e corresponding relative dissipation power; f normalized energy decrement for the numerical simulations in Figs. 15 and 16. Stability of the branches was not computed for this case same velocity $\left(\omega_{1} a_{1}=\omega_{2} a_{2}=\omega_{3} a_{3}=\omega_{4} a_{4}\right)$. In the figure, four peaks, corresponding to the four bumps of the SIM sections, are clearly recognizable. The highest peak is associated with the highest bump of $b_{4}$, while the lowest one is associated with the highest bump of $b_{1}$.

The three highest peaks occur at $\omega_{h} a_{h} \approx 0.4$, which means that by initiating the primary system slightly above this value the high dissipation power corresponding to the peaks is exploited. This explains the fast dissipation time obtained in this region. To facilitate the comparison, the same-colored regions adopted in Fig. 14a are depicted in Fig. 17b.

Another interesting aspect of the relative dissipation power curve in Fig. 17e is that, for $\omega_{h} a_{h}$ between 0.43 and 1.1, the $P_{\mathrm{r}}$ has five or more coexisting solutions. This explains why $C_{43}, C_{32}$ and $C_{21}$ coefficients are fuzzy for those values and a sharp limit above which the RCC is verified cannot be defined. In fact, it can be inferred that the dynamical response of the system depends on its exact initial conditions, and not only on the energy level. In spite of that, the dissipation time (Fig. 14a) experiences only relatively small variations, meaning that the different dynamical paths provide similar overall performance. From a design prospective, we remind that the oscillation amplitude was nondimensionalized with respect to the nonlinear stiffness of the NES; therefore, by adjusting the nonlinear stiffness coefficient, it is possible to define the energy level of optimal operation of the absorber.

\section{Conclusion}

In this study, the dynamics of a multi-degree-offreedom system equipped with a nonlinear energy sink (NES) are analyzed. Under impulsive excitation, it is shown that the NES is able to trigger different 
broadband energy dissipation mechanisms associated with a variety of modal energy exchanges; the latter are thoroughly investigated by resorting to the multidimensional slow invariant manifold (SIM). Interaction points lying on the SIM curves have been identified; they not only provide conditions at which multimodal interference occurs, but also enable to predict whether such interference is detrimental or not to overall energy dissipation. Sections of the SIM are effectively exploited to interpret the resonance capture cascade (RCC) phenomenon by allowing to track the evolution of the modal energy distribution. Based on the SIM, relative dissipation power curves are introduced in order to quantify the NES performance; it is shown that peaks on these curves correspond to optimal dissipation regimes. By discussing the dissipation performance, it is also highlighted that $\mathrm{RCC}$ regimes do not guarantee optimal conditions since they generally occur for energy levels higher than those at which the relative dissipation power modal tongues occur.

The main limit of the implementation of the SIM for NES performance estimation is that internal resonances are overlooked, while numerical simulations illustrate that they can significantly affect the dynamics. In particular, internal resonances seem to cause energy transfer from one mode of the primary system to a lower one via the NES. Another strong assumption adopted for the mathematical formalization of the SIM is that natural frequencies of the primary system are far from each other; however, closely spaced modes often occur in real systems and this might significantly affect the NES performance. These two aspects will be the subject of future studies.

Acknowledgements GH acknowledges the financial support of the Higher Education Excellence Program of the Ministry of Human Capacities in the frame of Biotechnology research area of Budapest University of Technology and Economics BIO) and Kevin Dekemele for fruitful discussions. FR acknowledges the financial support of PRIN 2015 N. 2015TTJN95.

Funding Open access funding provided by Budapest University of Technology and Economics.

\section{Compliance with ethical standards}

Conflicts of interest The authors declare that they have no conflict of interest.

Open Access This article is licensed under a Creative Commons Attribution 4.0 International License, which permits use, sharing, adaptation, distribution and reproduction in any medium or format, as long as you give appropriate credit to the original author(s) and the source, provide a link to the Creative Commons licence, and indicate if changes were made. The images or other third party material in this article are included in the article's Creative Commons licence, unless indicated otherwise in a credit line to the material. If material is not included in the article's Creative Commons licence and your intended use is not permitted by statutory regulation or exceeds the permitted use, you will need to obtain permission directly from the copyright holder. To view a copy of this licence, visit http://creativecommons.org/licenses/by/4.0/.

\section{References}

1. Vakakis, A.F., Manevitch, L., Gendelman, O., Bergman, L.: Dynamics of linear discrete systems connected to local, essentially non-linear attachments. J. Sound Vib. 264(3), 559-577 (2003)

2. Vakakis, A.F.: Shock isolation through the use of nonlinear energy sinks. Modal Anal. 9(1-2), 79-93 (2003)

3. Vakakis, A.F., McFarland, D.M., Bergman, L., Manevitch, L.I., Gendelman, O.: Isolated resonance captures and resonance capture cascades leading to single-or multi-mode passive energy pumping in damped coupled oscillators. J. Vib. Acoust. 126(2), 235-244 (2004)

4. Kerschen, G., Lee, Y.S., Vakakis, A.F., McFarland, D.M., Bergman, L.A.: Irreversible passive energy transfer in coupled oscillators with essential nonlinearity. SIAM J. Appl. Math. 66(2), 648-679 (2005)

5. Kerschen, G., Kowtko, J.J., McFarland, D.M., Bergman, L.A., Vakakis, A.F.: Theoretical and experimental study of multimodal targeted energy transfer in a system of coupled oscillators. Nonlinear Dyn. 47(1-3), 285-309 (2007)

6. Hubbard, S.A., McFarland, D.M., Bergman, L.A., Vakakis, A.F.: Targeted energy transfer between a model flexible wing and nonlinear energy sink. J. Aircr. 47(6), 1918-1931 (2010)

7. Sigalov, G., Gendelman, O., Al-Shudeifat, M., Manevitch, L., Vakakis, A.F., Bergman, L.: Resonance captures and targeted energy transfers in an inertially-coupled rotational nonlinear energy sink. Nonlinear Dyn. 69(4), 1693-1704 (2012)

8. Vakakis, A.F., Gendelman, O.V., Bergman, L.A., McFarland, D.M., Kerschen, G., Lee, Y.S.: Nonlinear Targeted Energy Transfer in Mechanical and Structural Systems, vol. 156. Springer, Berlin (2008)

9. Gendelman, O., Alloni, A.: Dynamics of forced system with vibro-impact energy sink. J. Sound Vib. 358, 301-314 (2015)

10. Bergeot, B., Bellizzi, S.: Asymptotic analysis of passive mitigation of dynamic instability using a nonlinear energy sink network. Nonlinear Dyn. 94(2), 1501-1522 (2018)

11. Bergeot, B., Bellizzi, S.: Steady-state regimes prediction of a multi-degree-of-freedom unstable dynamical system coupled to a set of nonlinear energy sinks. Mech. Syst. Signal Process. 131, 728-750 (2019)

12. Hurel, G., Ture Savadkoohi, A., Lamarque, C.-H.: Nonlinear vibratory energy exchanges between a two- 
degree-of-freedom pendulum and a nonlinear absorber. J. Eng. Mech. 145(8), 04019058 (2019)

13. Dekemele, K., De Keyser, R., Loccufier, M.: Performance measures for targeted energy transfer and resonance capture cascading in nonlinear energy sinks. Nonlinear Dyn. 93(2), 259-284 (2018)

14. Dekemele, K., Van Torre, P., Loccufier, M.: Performance and tuning of a chaotic bi-stable nes to mitigate transient vibrations. Nonlinear Dyn. 98(3), 1831-1851 (2019)

15. Habib, G., Romeo, F.: The tuned bistable nonlinear energy sink. Nonlinear Dyn. 89(1), 179-196 (2017)

16. Habib, G., Romeo, F.: "Comparative analysis of NES and TMD performance via high-dimensional invariant manifolds," In: Kovacic, I., Lenci, S. (eds.) IUTAM Symposium on Exploiting Nonlinear Dynamics for Engineering Systems, pp. 143-153, Springer (2018)

17. Starosvetsky, Y., Gendelman, O.: Interaction of nonlinear energy sink with a two degrees of freedom linear system: Internal resonance. J. Sound Vib. 329(10), 1836-1852 (2010)
18. Dekemele, K., Van Torre, P., Loccufier, M.: Design, construction and experimental performance of a nonlinear energy sink in mitigating multi-modal vibrations. J. Sound Vib. 473, 115243 (2020)

19. Hill, G.W.: On the part of the motion of the lunar perigee which is a function of the mean motions of the sun and moon. Acta Math. 8(1), 1-36 (1886)

20. Von Groll, G., Ewins, D.J.: The harmonic balance method with arc-length continuation in rotor/stator contact problems. J. Sound Vib. 241(2), 223-233 (2001)

21. Detroux, T.: Performance and Robustness of Nonlinear Systems Using Bifurcation Analysis. PhD thesis, Université de Liège, Liège, Belgium (2016)

Publisher's Note Springer Nature remains neutral with regard to jurisdictional claims in published maps and institutional affiliations. 